Journal of Astronomical Instrumentation, Vol. 8, No. 2 (2019) 1950001 (21 pages)

(C) The Author(s)

DOI: $10.1142 / \mathrm{S} 2251171719500016$

\title{
Adaptive Optics for Extremely Large Telescopes
}

\author{
Stefan Hippler \\ Max-Planck-Institut für Astronomie \\ Heidelberg 69117, Germany \\ hippler@mpia.de
}

Received May 7, 2018; Revised August 7, 2018; Accepted September 26, 2018; Published November 2, 2018

\begin{abstract}
Adaptive Optics (AO) has become a key technology for the largest ground-based telescopes currently under, or close to beginning of, construction. AO is an indispensable component and has basically only one task, that is to operate the telescope at its maximum angular resolution, without optical degradations resulting from atmospheric seeing. Based on three decades of experience using AO usually as an add-on component, all extremely large telescopes and their instrumentation are designed for diffraction limited observations from the very beginning. This paper illuminates various approaches of the ELT, the Giant Magellan Telescope (GMT), and the Thirty-Meter Telescope (TMT), to fully integrate AO in their designs. The paper concludes with a brief look into the requirements that high-contrast imaging poses on AO.
\end{abstract}

Keywords: Adaptive optics, ELT, TMT, GMT, high-contrast imaging, review.

\section{Introduction}

Over the past 30 years, ground-based astronomical observations have drawn level with their spacebased counterparts. Thanks to adaptive optics (AO), the seeing limit caused by optical turbulence of the Earth atmosphere is decreasingly a real limitation for existing large optical telescopes. For the three extremely large telescopes (ELTs) currently under construction or about to begin construction, $\mathrm{AO}$ is an integral part of telescope design, allowing to achieve the highest angular resolution on sky: the telescope's diffraction limit.

\subsection{Basic principle}

The technique behind AO was first formulated by American astronomer Horace W. Babcock (1953) and can be summarized as canceling optical aberrations caused by the atmosphere in real-time.

This is an Open Access article published by World Scientific Publishing Company. It is distributed under the terms of the Creative Commons Attribution 4.0 (CC-BY) License. Further distribution of this work is permitted, provided the original work is properly cited.
Babcock's proposal to compensate astronomical seeing was to measure wavefront distortions (using a rotating knife-edge) and feedback that information to a wavefront correction element (Eidophor mirror). In modern applications, a fast real-time controller (RTC) sits in between a wavefront sensor (WFS) and a wavefront corrector (e.g. a deformable mirror (DM)) such that these three elements build a closed-loop system, i.e. controlling wavefronts before they reach the telescope instrument (e.g. the scientific camera). However, it took until the early 1970s before Babcock's idea could be tested experimentally (Hardy et al., 1977).

\subsection{Early years of $A O$ in astronomy using single natural and laser guide stars}

The usage of AO in routine astronomical observations on telescopes of the $3-4 \mathrm{~m}$ class can be dated back to the end of the 1980s when the instrument Come-On (Rousset et al., 1990) delivered diffraction limited images in the near-infrared spectral range on a $1.5 \mathrm{~m}$ telescope. Upgrades from Come-On to Come-On-Plus (Gendron et al., 1991) and eventually to ADONIS (Beuzit et al., 1994) allowed 


\section{S. Hippler}

diffraction limited imaging on a $3-4 \mathrm{~m}$ class telescope in the $1-5 \mu \mathrm{m}$ spectral range.

Around 10 years later, at the end of the 1990s, the first AO system on a 8-10 m class telescope - the Keck II telescope - began operation (Wizinowich et al., 2000).

First laser guide star (LGS) assisted AO systems, which were installed during the 1990s on smaller telescopes, can be seen in retrospect as prototypes, even though few scientific results have been published (Davies et al., 1999; Hackenberg et al., 2000). LGSs boost the operational readiness of AO-assisted observations of faint objects. An overview and historical review of sodium LGSs can be found in d'Orgeville \& Fetzer (2016). LGS systems using other wavelengths than the sodium one at $589 \mathrm{~nm}$, are usually called Rayleigh LGS systems, and for example the first Rayleigh system was built and used by the US military in the early 1980s (Fugate et al., 1991).

On $8 \mathrm{~m}$ class telescopes, sufficiently bright LGS AO systems can increase the low sky coverage obtained with natural guide star (NGS) AO systems from a few percent up to about $80 \%$, depending on the criterium for sky coverage. The most simple criterium for the sky coverage of an $\mathrm{AO}$ system is that the $\mathrm{AO}$ improves the natural seeing. This gives the highest sky coverage values. A much more stringent criterium is that the AO shall deliver fully diffraction limited point spread functions with long exposure Strehl numbers $>80 \%$ at the observing wavelength. NGS AO sky coverage with sufficiently bright guide stars depends on the isoplanatic angle, while LGS AO sky coverage depends on focal and tilt anisoplanatism (van Dam et al., 2006). These limitations are due to atmospheric anisoplanatism that can be partially eliminated with the usage of multiple guide stars, either natural, artificial or a combination of both.

\subsection{Wide-field and extreme $A O$}

A first attempt with multiple NGSs took place on the $8 \mathrm{~m}$ VLT on Paranal observatory in Chile in 2007. Implemented in an AO type named multiconjugate AO (MCAO) was the MCAO demonstrator instrument MAD (Marchetti et al., 2008). Results with this instrument, cf. Campbell et al. (2010); Meyer et al. (2011), showed that reconstructing and correcting wavefronts over large field of views using multiple DMs and WFSs matched the predictions. Compared to - nowadays sometimes called classical AO - single conjugate AO
(SCAO) systems with a typical non-homogeneous corrected field of view of a few 10 arcsec, MAD delivered a 2 arcmin wide homogeneously corrected field of view.

About four years later in 2011, the Gemini MCAO system GeMS, the first multiple laser and NGS AO system saw first light on Cerro Pachón in Chile (Rigaut et al., 2014; Neichel et al., 2014). It is currently the only operational facility instrument of its kind. GeMS has an estimated sky coverage of almost $100 \%$ in the galactic plane, $>35 \%$ at medium galactic latitudes, and $\sim 20 \%$ at the galactic poles (Marin et al., 2017), limited mainly by the required three NGSs for measuring tip and tilt aberrations over the $85^{\prime \prime} \times 85^{\prime \prime}$ corrected field of view.

Another prediction of MCAO in combination with a tomographic wavefront reconstruction over the entire observing volume in the atmosphere (Ragazzoni, 1999; Ragazzoni et al., 2000) was the sky coverage boost to almost $100 \%$ of the sky for an up to $100 \mathrm{~m}$ sized future optical telescope (Gilmozzi et al., 1998) with NGSs only. Nevertheless, for the currently planned MCAO systems on the $39 \mathrm{~m}$ ELT and the $30 \mathrm{~m}$ TMT (Sec. 2), both natural and LGSs are foreseen. The expected/required sky coverage for the TMT MCAO instrument is above $70 \%$ for median seeing conditions and close to $100 \%$ under $25 \%$ best seeing conditions (Wang et al., 2012). In terms of image quality, under nominal conditions, MCAO systems deliver lower Strehl ratios compared to SCAO systems.

Another type of wide field $\left(\geq 2^{\prime} \times 2^{\prime}\right.$ up to 10 $\left.20^{\prime} \times 10-20^{\prime}\right)$ AO system, which delivers a corrected image image quality between seeing limited performance and MCAO performance, are so called ground-layer adaptive optics (GLAO) systems or "seeing improvers". Proposed by François Rigaut in 2002 (Rigaut, 2002), GLAO only corrects the optical turbulence located within the first $\approx 500-1000 \mathrm{~m}$ above the telescope pupil. The performance depends on the amount of optical turbulence within the ground layer compared to the total atmospheric optical turbulence. Using multiple NGSs, first results obtained with a GLAO system on $8 \mathrm{~m}$ class telescopes were achieved in 2007 (Marchetti et al., 2007). Using a 5 Rayleigh LGSs GLAO system on the $6.5 \mathrm{~m}$ MMT telescope in Arizona (Hart et al., 2010 ), the uncorrected seeing at $2.2 \mu \mathrm{m}$ of $0.61^{\prime \prime}$ was improved to $0.22^{\prime \prime}$, about a factor of 3 . Results (see Fig. 1) obtained with a 3 Rayleigh LGSs GLAO system on the $8.4 \mathrm{~m}$ large binocular telescope (LBT) 

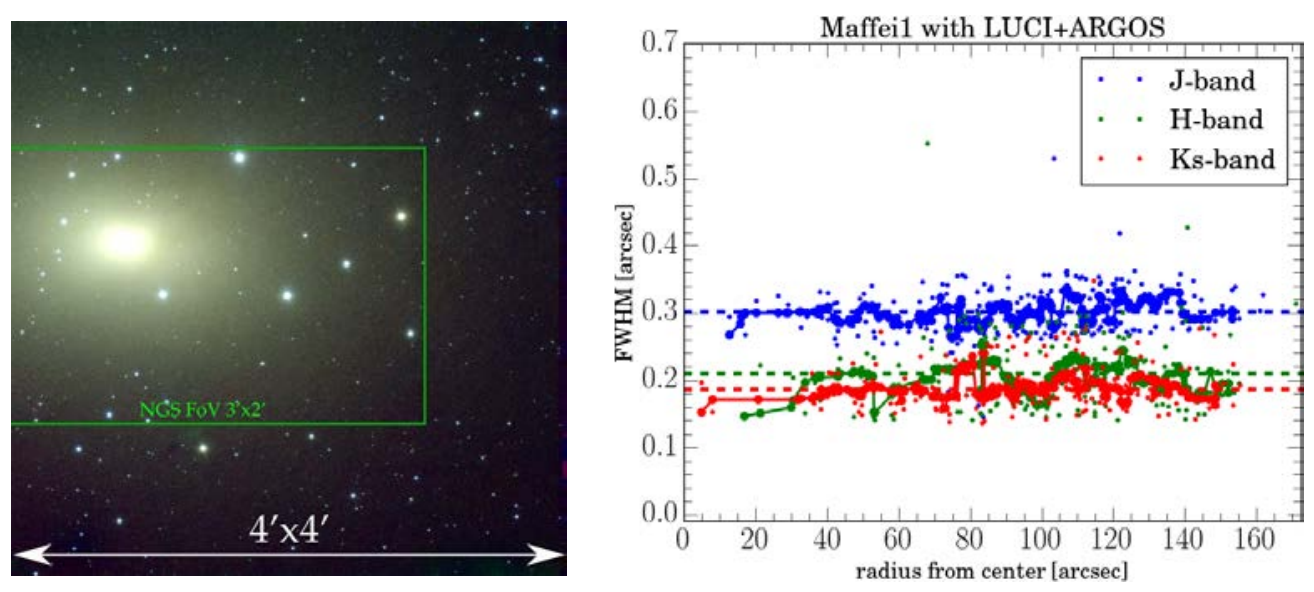

Fig. 1. Exemplary GLAO performance at the LBT with Rayleigh LGSs. (Left) LBT LUCI+ARGOS observation of the elliptical galaxy Maffei1 in J, H and $\mathrm{K}^{s}$ broad band for a total of 7.5 min exposure time. The field of view (FoV) spans $4^{\prime} \times 4^{\prime}$ and shows a uniform resolution all over. The field-of-view available to pick a NGS for tip-tilt and truth sensing is highlighted in green. (Right) Point-spread function full width at half maximum (FWHM) as a function of radius from the center of the FoV, where was also NGS for tip-tilt sensing. The constant distribution shows that there is no apparent sign of anisoplanatism and that the GLAO correction is very uniform. Figures and slightly modified caption are taken from Orban de Xivry et al. (2016).

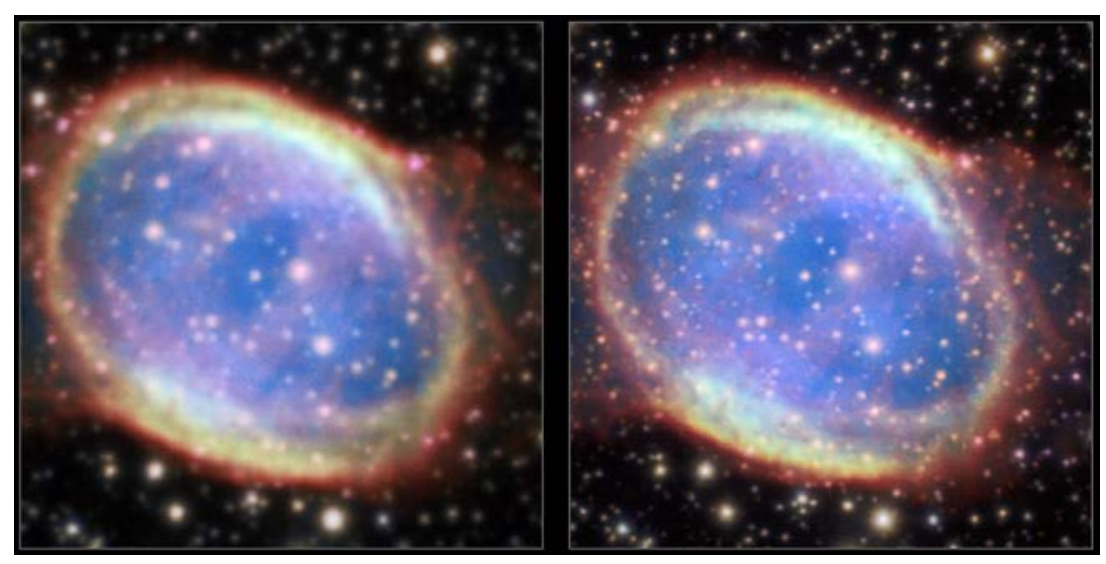

Fig. 2. Exemplary GLAO performance at the very large telescope (VLT) with sodium LGSs. VLT MUSE+AOF view of the planetary nebula NGC 6563 without GLAO correction (left) and with GLAO correction using 4 sodium LGSs (right). The field of view is $1.01 \times 1.03$ arcmin. Both images are color composites observed in the optical with various band filters ranging from $500 \mathrm{~nm}$ to $879 \mathrm{~nm}$. (ESO, 2017).

in Arizona (Orban de Xivry et al., 2016) show that a seeing improvement by a factor of 2 can be achieved under good conditions, down to $0.2^{\prime \prime}$ at $1.6 \mu \mathrm{m}$ over a 4 arcmin wide field of view.

GLAO systems using multiple sodium LGSs are currently installed and commissioned on Paranal (Paufique et al., 2016; La Penna et al., 2016). First results obtained with the adaptive optics facility (AOF) and the instrument MUSE - good to recognize in (Fig. 2) - show the potential of this technique in particular for wide-field GLAO spectroscopic observations. During the MUSE science verification in 2017, observations under one arcsecond seeing conditions were improved with GLAO down to $\sim 0.6$ arcsec across the full 1 arcmin field of view (Leibundgut et al., 2017).

The planning and development of second generation AO systems for $8-10 \mathrm{~m}$ class telescopes, often in the context of exoplanet characterization in combination with high-contrast imaging (Milli et al., 2016), started at the beginning of the 21st century (Gratton et al., 2004; Fusco et al., 2005, 2006; Beuzit et al., 2006; Feldt et al., 2007; Esposito et al., 2006; Macintosh et al., 2006; Graham et al., 2007; Martinache \& Guyon, 2009; Guyon et al., 2010). First science results with instruments based on these 


\section{S. Hippler}
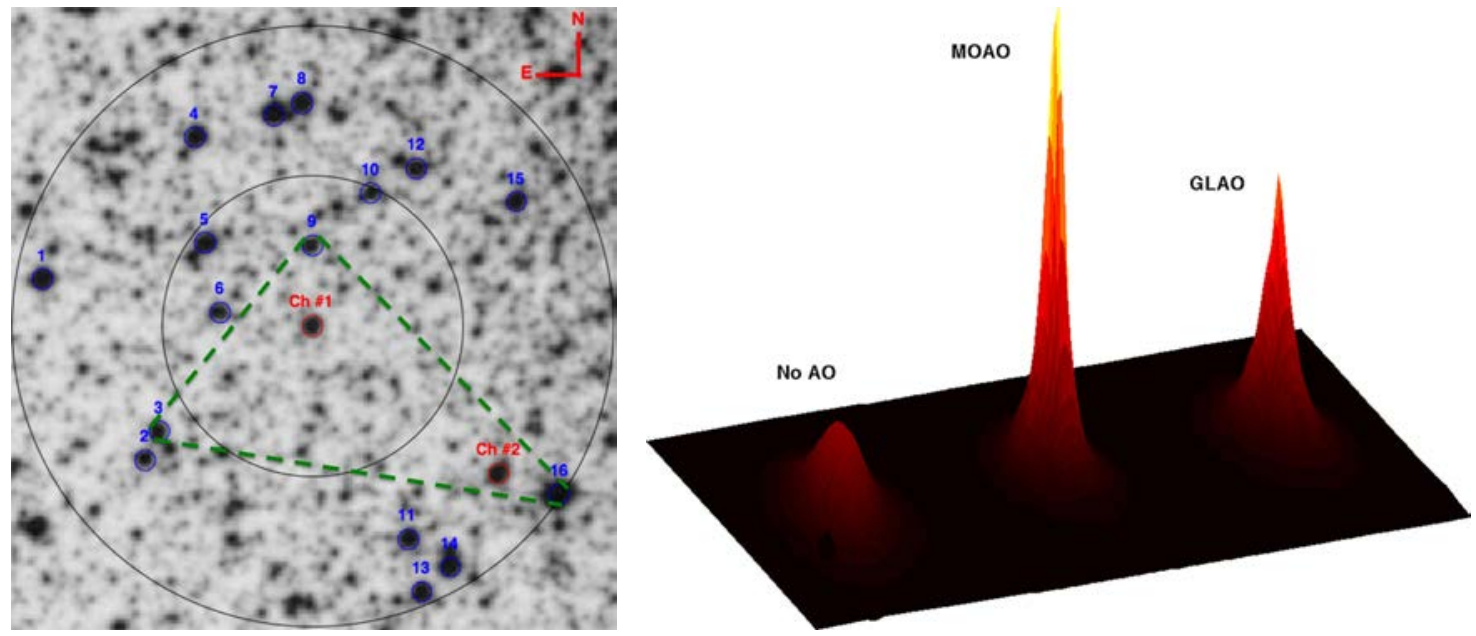

Fig. 3. Exemplary RAVEN asterism inside the M22 globular cluster (left). The two 4 arcsec $\times 4$ arcsec regions, science channel $(\mathrm{Ch}) \# 1$ and $(\mathrm{Ch}) \# 2$ lie in the green colored triangle formed by three NGSs, each feeding its own WFS. Further suitable guide stars are marked with blue circles. The diameter of the two black circles correspond to 60 and 120 arcsec. The right figure shows an exemplary PSF of a M22 star with no AO (left), and with MOAO and GLAO corrections (middle and right respectively). MOAO is shown to outperform GLAO. Both figures were taken from Lamb et al. (2017).

extreme $\mathrm{AO}(\mathrm{XAO})$ systems were achieved around 10 years later (Esposito et al., 2010, 2013; Macintosh et al., 2015; Vigan et al., 2016; Maire et al., 2016; Currie et al., 2017). Typically, AO systems tagged "extreme" sample wavefronts at kilohertz frame rates, and correct wavefronts with a high number of actuators (for example: 672 actuators in the LBT first light AO system (Esposito et al., 2006) or 4096 actuators available in the Gemini planet imager AO system (Macintosh et al., 2006)).

The last flavor of AO type to mention before looking at the AO-related hardware designs for the upcoming ELTs, is the multi-object adaptive optics (MOAO) system. The goal of MOAO is to compensate atmospheric turbulence over a wide field of view up to $5-10$ arcmin, using individual DMs for each object to be observed. Using a small number of either natural or LGSs, similar to MCAO, the wavefront is reconstructed using tomographic methods (Ragazzoni et al., 1999; Vidal et al., 2010). Each DM is optimally shaped to correct the turbulence in its viewing direction, like a multi-SCAO system operated in open-loop. MOAO demonstrator systems used in the laboratory (Laag et al., 2008; Ammons et al., 2008) and on sky (Gendron et al., 2011; Lardière et al., 2014), have shown that MOAO performs close to conventional SCAO systems.

The first MOAO system installed on a $8 \mathrm{~m}$ class telescope was the RAVEN (Andersen et al., 2012; Lardière et al., 2014; Davidge et al., 2015; Ono et al.,
2016; Lamb et al., 2017) demonstrator, which was in operation at the Subaru telescope between 2014 and 2015. Developed as a pathfinder for the $30 \mathrm{~m} \mathrm{TMT}$, results obtained with this instrument are encouraging, with better performance than GLAO (Fig. 3) but not reaching SCAO performance. RAVEN can use either three NGSs or a combination of one sodium LGS and two NGSs. Two DMs with 145 actuators each, allow AO corrections on two science fields with a maximum separation of about 3.5 arcmin.

Conceptual drawings of the various AO concepts, i.e. SCAO, GLAO, MCAO, LTAO, and MOAO, including drawings of conventional WFSs and photos of DMs, can be found in the review paper of Davies \& Kasper (2012).

It goes without saying that the current, often already the second generation of AO systems on 8-10 m class telescopes, has generated a wealth of experience. These experiences and lessons learned (see for example Lloyd-Hart et al. (2003); Wizinowich (2012); Fusco et al. (2014); Lozi et al. (2017)) are of course incorporated into the design and simulations of the AO facilities to be built. Since it would go beyond the scope of this review, here are just two current examples.

The SPHERE XAO system installed at the Paranal observatory discovered a so called low wind effect Milli et al. (2018) (sometimes also named island effect), which basically distorts the wavefronts in the pupil of large telescopes obstructed by 
spiders. The effect occurs mainly when wind speeds above the telescope are close to zero. The SPHERE $\mathrm{XAO}$ system cannot measure these piston aberrations, thus they propagate uncorrected to the science channel and degrade the image quality. The solutions proposed and tested will influence the design of possible upgrades to the SPHERE XAO system as well as future AO systems.

The second example concerns a general problem of most $\mathrm{AO}$ systems: telescope vibrations and their impact on AO performance. Experiences with the SCExAO system at the Subaru telescope (Lozi et al., 2016) show weaknesses of the control system in handling vibrations, which may be mitigated by the use of predictive-control (Males \& Guyon, 2018).

\section{ELT, GMT, and TMT AO Systems}

\subsection{AO architecture and types for first light instrumentation}

All ELTs currently under design and construction have AO kind of built-in. This means that either all components of the $\mathrm{AO}$ are part of the telescope or that the AO pieces are split among telescope and instruments.

The $39 \mathrm{~m}$ European Southern Observatory's (ESO) ELT (previously E-ELT) on Cerro Armazones in Chile has a five-mirror optical design. Its large adaptive quaternary mirror (Fig. 4) has about 5,000 actuators.

The Gregorian-type $25.4 \mathrm{~m}$ Giant Magellan Telescope (GMT) at Las Campanas observatory in Chile, developed by a United-States led consortium,

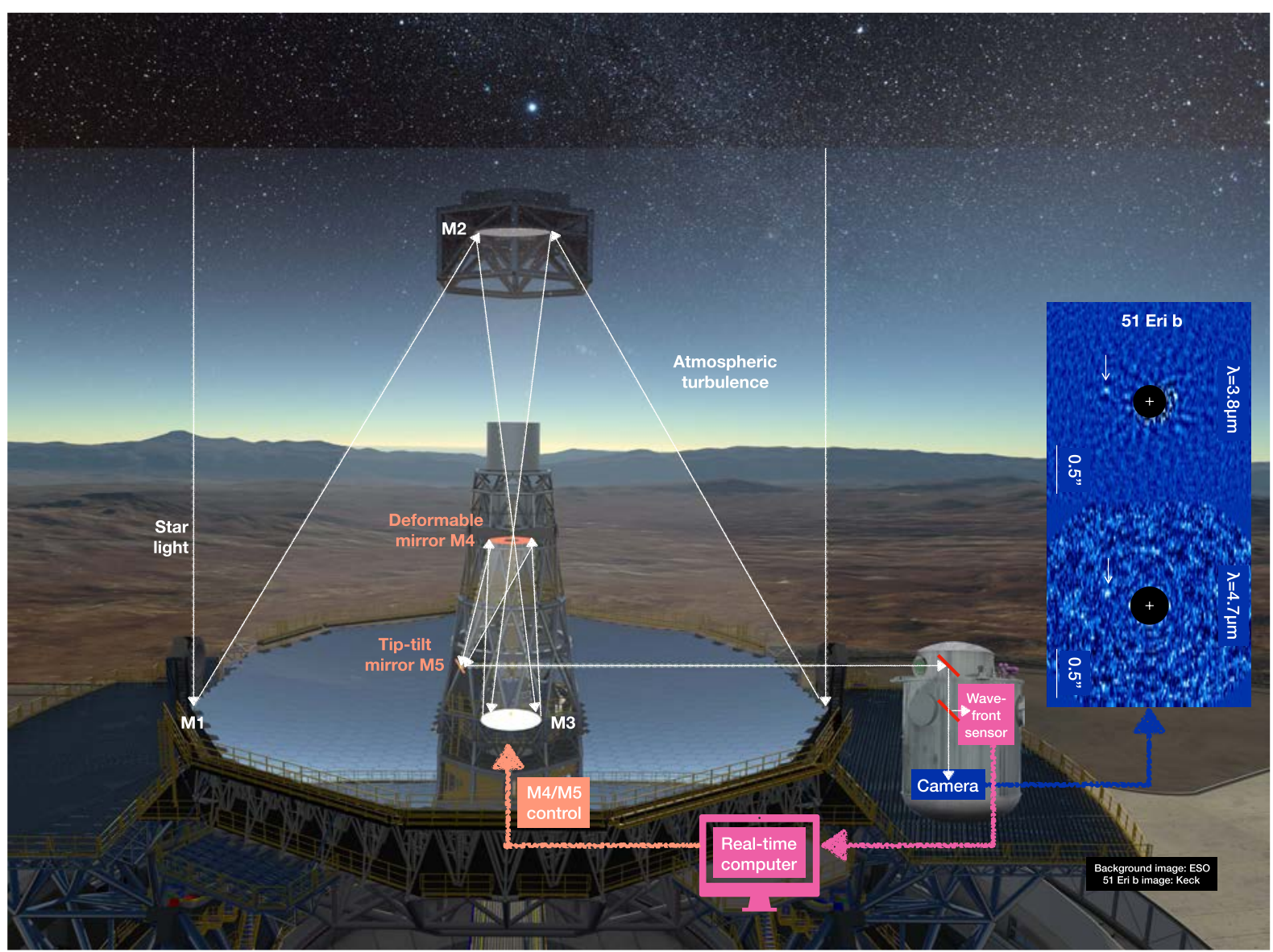

Fig. 4. Single-conjugate AO scheme for the ELT METIS instrument. Atmospheric turbulence distorts the observed starlight collected by the ELT's segmented primary mirror M1. Reflected up to mirror M2 and down to mirror M3, passing through a hole in the DM M4, the last reflection on the tip-tilt mirror M5 forwards the light into the METIS cryostat on the right, outside of the telescope. The METIS internal WFS measures the atmospheric distortions. A real-time computer estimates the actual wavefront and delivers the corresponding information to properly shape the DM M4 as well as to properly steer the tip-tilt mirror M5 with the M4/M5 control unit. Eventually, an exoplanet like 51 Eri b can be detected at two different wavelengths (see blue-colored inset on the right). 


\section{S. Hippler}
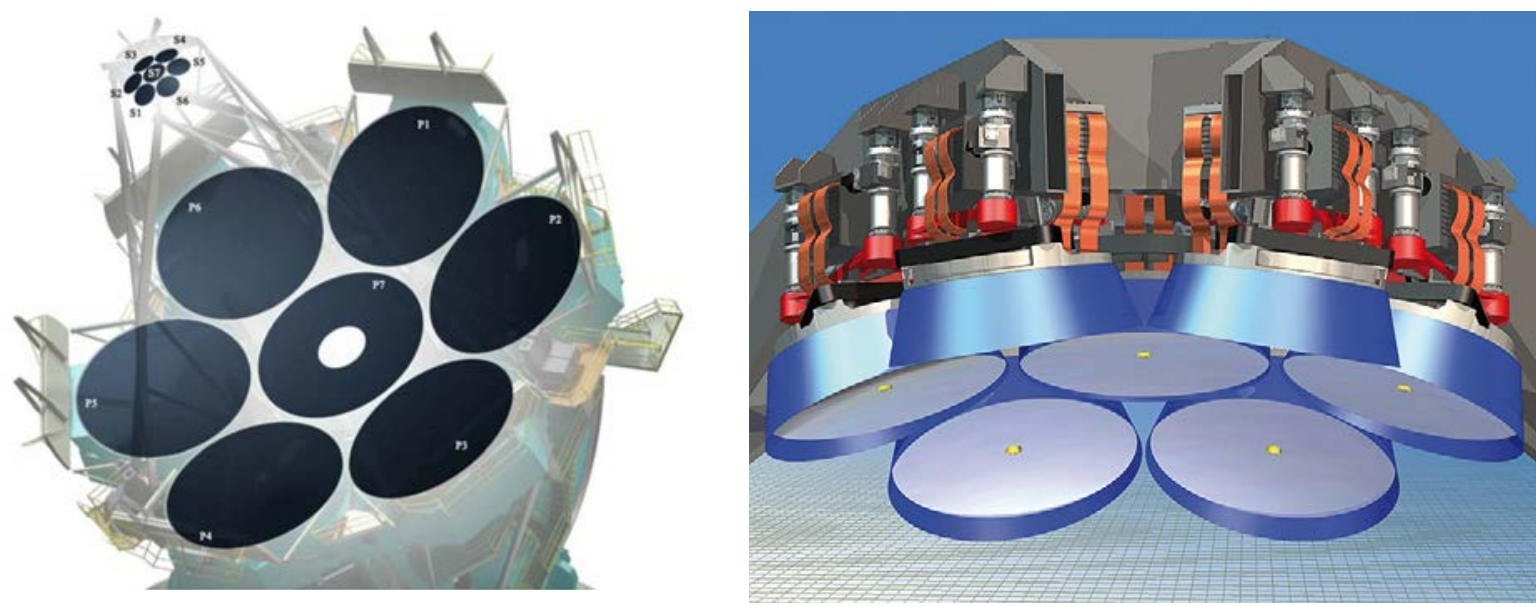

Fig. 5. GMT optical layout of the seven segment primary (P1-P7) and secondary mirror (S1-S7) on the left, a more detailed rendering of the seven segment adaptive secondary on the right. Each adaptive secondary segment has 672 contactless voice-coil actuators. Figures taken from GMTO (2013).

will have a large segmented adaptive secondary mirror (Fig. 5) with roughly the same number of actuators as the ELT.

The Thirty Meter Telescope (TMT), which will be located either on Mauna Kea, Hawaii or Canary Islands, Spain, is developed by a consortium of universities, foundations, and national observatories in the United States, Canada, China, India, and Japan. It has a Ritchey-Chrétien optical design and no adaptive mirror built-in. The TMT will probably start operation with a conventional $3 \mathrm{~m}$ class "active" secondary mirror and a facility AO device including DMs and WFSs. This AO facility, called NFIRAOS (see Sec. 2.2), includes DMs with about 8000 actuators in total, and will feed three instruments.

\subsubsection{TMT first-generation instruments}

First-generation instruments of the TMT using AO are infrared imager and spectrograph (IRIS) (Larkin et al., 2016) and infrared multi-slit spectrograph (IRMS) (Mobasher et al., 2010).

IRIS is a first-generation near-infrared (0.84$2.4 \mu \mathrm{m})$ instrument being designed to sample the diffraction limit of the TMT. IRIS will include an integral field spectrograph $(\mathrm{R}=4000-10,000)$ and imaging camera $\left(34^{\prime \prime} \times 34^{\prime \prime}\right)$. Both the spectrograph and imager will take advantage of the high spatial resolution achieved with the Narrow-Field Infrared Adaptive Optics System (NFIRAOS) at four spatial scales $\left(0.004^{\prime \prime}, 0.009^{\prime \prime}, 0.025^{\prime \prime}, 0.05^{\prime \prime}\right)$. The features of the design will enable a vast range of science goals covering numerous astrophysical domains including: solar system science, extrasolar planet studies, star formation processes, the physics of super-massive black-holes and the composition and formation of galaxies, from our local neighborhood to high-redshift galaxies. Description for IRIS taken from TMT (2017b) and OIR-Laboratory-UCSD (2017).

The IRMS is a near diffraction-limited multi-slit near-infrared spectrometer and imager. It will be fed by NFIRAOS. IRMS will provide near-infrared imaging and multi-object spectroscopy in the spectral range of $0.97-2.45 \mu \mathrm{m}$. The science case for IRMS includes planetary astronomy (exoplanets), stellar astronomy (massive stars, young star clusters), active galactic nuclei (co-evolution of black holes and galaxies), inter-galactic medium (interaction with galaxies at high-z), and the high-redshift universe (population III stars, cosmic re-ionization, nature of the high-z galaxies). Description for IRMS is taken from TMT-UCR (2014).

\subsubsection{ELT first-generation instruments}

First-generation instruments of the ELT are HARMONI (Thatte et al., 2016), MAORY (Diolaiti et al., 2016), METIS (Brandl et al., 2016), and MICADO (Davies et al., 2016). The short descriptions of these instruments, enclosed in quotation marks below, are taken from ESO (2018).

HARMONI, the high angular resolution monolithic optical and near-infrared integral field spectrograph, "will be used to explore galaxies in the early Universe, study the constituents of the local 
Table 1. Type of AO planned for first light instrumentation. List of used abbreviations. ${ }^{\mathrm{c}-\mathrm{f}}$

Telescope and instrument $\rightarrow$ AO type $\downarrow$

\begin{tabular}{ll}
\hline $\mathrm{SCAO}^{\mathrm{a}}$ & METIS, HARMONI, MICADO \\
$\mathrm{MCAO}^{\mathrm{a}, \mathrm{b}}$ & MICADO-MAORY \\
$\mathrm{LTAO}^{\mathrm{b}}$ & HARMONI \\
$\mathrm{GLAO}^{\mathrm{a}}$ & \\
$\mathrm{MOAO}$ & MOSAIC \\
& $($ Morris et al. (phase A study \\
\end{tabular}

Giant Magellan telescope (GMT), $24.5 \mathrm{~m}$

Notes: ${ }^{\mathrm{a}}$ Using NGSs. ${ }^{\mathrm{b}}$ Using sodium LGSs.

${ }^{\mathrm{c}}$ GLAO: ground-layer AO, LTAO: laser tomography AO, MCAO: multi-conjugate AO, MOAO: multi-object AO, SCAO: singleconjugate AO.

${ }^{\mathrm{d}}$ HARMONI: high angular resolution monolithic optical and near-infrared integral field spectrograph, MAORY: multi-conjugate adaptive optics relay, METIS: mid-infrared ELT thermal imager and spectrograph, MICADO: multi-adaptive optics imaging camera for deep observations, MOSAIC: multi-object spectrograph for Astrophysics, inter galactic medium, and Cosmology.

${ }^{\text {e}}$ G-CLEF: GMT consortium large earth finder, GMTIFS: GMT integral-field spectrograph, GMACS: GMT multi-object astronomical and cosmological spectrograph, GMTNIRS: GMT near-infrared spectrograph.

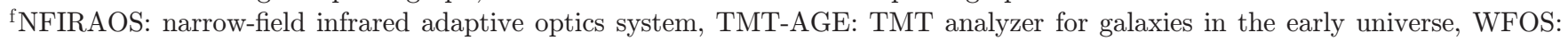
wide-field optical spectrometer.

Universe and characterize exoplanets in great detail."

METIS, the mid-infrared imager and spectrograph, will "focus on five scientific goals: exoplanets, proto-planetary disks, Solar System bodies, active galactic nuclei, and high-redshift infrared galaxies."

MICADO, the multi-AO imaging camera for deep observations, "is the first dedicated imaging camera for the ELT. MICADO's sensitivity will be comparable to the James Webb Space Telescope, but with six times the spatial resolution."

MAORY, the multi-conjugate AO relay for the ELT, "is designed to work with the imaging camera MICADO and with a second future instrument. MAORY will use at least two DMs, including the $\mathrm{DM}$ of the telescope. It measures the light from a configuration of six sodium LGSs, arranged in a circle on the sky, to obtain a kind of three-dimensional mapping of the turbulence. The laser guide stars are projected from around the circumference of the telescope's primary mirror."

\subsubsection{GMT first-generation instruments}

For the GMT, the suite of first-light instruments (Jacoby et al., 2016) using AO consists of a fiberfed, cross-dispersed echelle spectrograph able to deliver precision radial velocities to detect low-mass exoplanets around solar-type stars, hence the name of the instrument GMT-consortium large earth finder G-CLEF (Szentgyorgyi et al., 2016). Both, the wide-field optical multi-object spectrograph GMACS (DePoy et al., 2012) and G-CLEF will benefit from the GMT's ground-layer AO observing mode (Bouchez et al., 2014; van Dam et al., 2014). The near-infrared integral field spectrograph and imager GMTIFS (Sharp et al., 2016) and the nearinfrared high-resolution spectrograph GMTNIRS (Jaffe et al., 2016; Jacoby et al., 2016) will use the GMT's laser tomography AO system (Bouchez et al., 2014; van Dam et al., 2016).

An overview of all three telescopes, their AO type, and their planned first suite of instrumentation is given in Table 1.

\subsection{Wavefront sensing set-up}

\subsubsection{Natural and artificial laser guide stars}

WFSs analyze light passing through the Earth's atmosphere. The ideal light source for wavefront sensing, called NGS, is a stellar point like object, as close as possible to the scientific target, and sufficiently bright to minimize measurement errors. Artificial light sources created high above the telescope - for example in the $\sim 100 \mathrm{~km}$ high sodium layer of the mesosphere - , called LGSs, can be positioned as close as required to the scientific target. Their brightness depends on the used technology. LGSs enormously increase the usability of AO systems. LGSs as well as the technique of laser tomography AO are mentioned in this article but going into details is beyond the scope of this review. 


\section{S. Hippler}

Table 2. Planned LGS facilities on ELT, GMT, and TMT.

\begin{tabular}{|c|c|c|c|}
\hline Telescope & Laser system & $\begin{array}{l}\text { Power per } \\
\text { laser [Watt] }\end{array}$ & Comments \\
\hline ELT & sodium laser $\mathrm{CW}$ & $\approx 22$ & $\begin{array}{l}\text { At least four lasers based on the VLT four LGSF (Bonaccini } \\
\text { Calia et al., 2014). }\end{array}$ \\
\hline GMT & sodium laser & $\approx 20$ & $\begin{array}{l}\text { Six lasers for LTAO observations, D'Orgeville et al. (2013); } \\
\text { Bouchez et al. (2014) }\end{array}$ \\
\hline TMT & sodium laser $\mathrm{CW}$ or pulsed & $20-25$ & Up to nine lasers, Li et al. (2016) \\
\hline
\end{tabular}

For an overview, Table 2 summarizes for each telescope the plans for installing LGS facilities.

\subsubsection{Wavefront sensing architecture}

The GMT SCAO and LTAO architecture (Fig. 6) is designed to use reflected light from a tilted instrument entrance window for wavefront sensing, using either light from natural or LGSs. The reconstructed wavefront is used to shape the adaptive secondary mirror of the GMT, such delivering corrected wavefronts to the instrument. In the case of LGS wavefront sensing additional on-instrument WFSs are required to sense low-order atmospheric aberrations. For GLAO operations, the GMT has an integrated acquisition, guiding, and wavefront sensing system (AGWS) analyzing the light in an annular field of view outside the science instruments field of view. This "technical" field of view with an inner diameter of about 6 arcmin and an outer diameter of about $10 \mathrm{arcmin}$, is large enough to find suitable NGSs for GLAO operations.

In contrast to the GMT, the ELT does all AO related wavefront sensing inside the instruments. An exception from this ELT AO design is MAORY, which is designed to support two instruments. Wavefront compensation is performed using the ELT's internal adaptive mirrors M4 and M5 as shown in Fig. 4. Active optics and possible GLAO support is done in a similar way as for the GMT, i.e. using a "technical" field of view.

How does the TMT compare with the GMT or ELT AO architecture? The TMT has a facility MCAO/SCAO system called NFIRAOS (Herriot et al., 2014, 2017), which can feed three

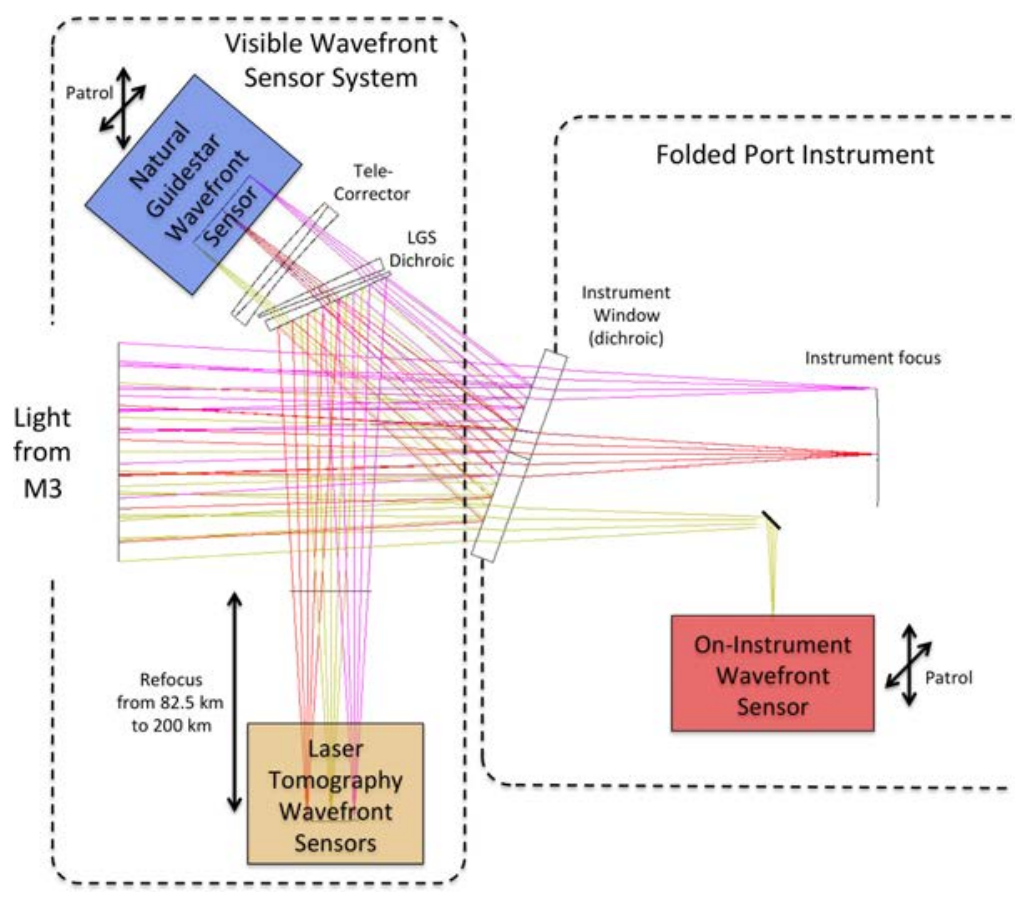

Fig. 6. The GMT LTAO, and SCAO design. Light reflected from the telescope's 3rd mirror (M3), before entering the science instrument, passes through the wavefront sensing area of the telescope. Figure taken from Bouchez et al. (2014). 
instruments: an infrared imaging spectrograph IRIS, an infrared multi-slit spectrometer IRMS, and a third future instrument. From this perspective, NFIRAOS is similar to the ELT's MAORY "relay" system, which feeds MICADO and a second future instrument. In its current design, the TMT itself has no adaptive DM built-in, instead, the wavefront correction devices are located inside NFIRAOS. A laser guide star facility (LGSF) will provide at least six sodium LGSs (Li et al., 2016) feeding NFIRAOS, thus enabling LGS MCAO observations (Boyer \& Ellerbroek, 2016). In September 2017, a design study for an adaptive secondary mirror was launched (TMT, 2017a). In particular, the wide-field optical spectrometer (WFOS) would benefit from such an integrated wavefront correction unit, as it is not connected to the NFIRAOS unit, and the LGSF is designed to provide various asterisms, including a GLAO asterism TMT (2018a).

Table 3 summarizes the main characteristics of the WFS used with the first light and first-generation respectively instrumentation.

\subsection{Wavefront correction devices}

This brings us to the topic of wavefront correction devices, their characteristics and location within each of the described telescopes and instruments, respectively. As shown in Fig. 4, the ELT comes with a DM M4 and a fast steering mirror M5. The $2.4 \times 2.5 \mathrm{~m}$ sized elliptic mirror M4 is supported by 5316 contactless voice-coil actuators (Biasi et al., 2016). This mirror can be controlled at frequencies up to $1 \mathrm{kHz}$. Together with the fast and flat steering mirror M5, which compensates for rather large amplitude atmospheric tip-tilt aberrations (field stabilization), this combination in interplay with the wavefront sensing devices, allows GLAO, SCAO, and LTAO observations. The ELT's MCAO relay MAORY adds another two DMs for instruments attached to it. Each of them with 500-1000 actuators (actuator number and technology not yet decided).

The GMT has two exchangeable secondary mirrors, each $3.25 \mathrm{~m}$ in diameter, segmented and shaped similar to the primary mirror (Fig. 5). The fully adaptive secondary mirror (ASM) is supported by 4704 contactless voice-coil actuators. An alternative fast steering secondary mirror (FSM) with seven rigid circular segments (Lee et al., 2017) will be used during commissioning of the telescope and later on during ASM maintenance or repair.
Wavefront correction at the TMT takes place in it's NFIRAOS facility (Fig. 7) operated at a temperature of -30 degrees. A tip-tilt stage and two DMs with a total number of 7673 actuators (3125 actuators for the ground layer DM and 4548 actuators for the high-layer DM) support MCAO observations. As mentioned in Sec. 2.2, TMT organization has launched a design study for an adaptive secondary mirror in September 2017.

Table 4 summarizes some basic parameters of the planned wavefront correction elements for all three telescopes. From today's perspective, piezo actuator technology is still in use, voice-coil actuator technology has become a standard, and bimorph mirror technology has disappeared. Liquid crystal spatial light modulators do not play a role in the context of $\mathrm{AO}$ for the first light instrumentation of the three telescopes. The role of micro electro-mechanical systems (MEMS) based DMs is rather small, only the GMT considers a MEMS DM for some of their first light instrumentation (Copeland et al., 2016).

A further increase in actuator density, i.e. for future XAO systems, from $5-10$ actuators $/ \mathrm{m}^{2}$ to 12.5-25 actuators $/ \mathrm{m}^{2}$ seems possible but has to be developed (Madec, 2012; Riaud, 2012; Kasper et al., 2013; Kopf et al., 2017). For high-contrast XAO systems, high-density DMs might be used to remove residual speckles seen in the science focal plane. In combination with the science focal plane detectors behind coronagraphs, a second stage AO system can be set-up in order to calibrate and remove residual speckles seen in the science images (Macintosh et al., 2006; Thomas et al., 2015).

\subsection{AO real-time control systems}

The last major component of an AO system is the real-time control system (RTC), which receives wavefront measurements from the WFSs, calculates wavefront estimates, and eventually sends control signals to the wavefront correction devices like DMs and tip-tilt mirrors. For the SCAO case, the largest size of an interaction matrix using numbers from Tables 3 and 4 has about 5000 actuators and 10,000 wavefront $x-y$ gradients.

The time available due to AO latency requirements, i.e. the time to

- receive pixel data from the WFSs,

- pre-process the raw WFS data, 


\section{S. Hippler}

Table 3. WFSs planned for first light instrumentation of ELTs. ${ }^{\mathrm{f}}$ [SHS: Shack-Hartmann WFS. Pyramid: Pyramid wavefront sensor. QC: Quad-cell detector.]

\begin{tabular}{|c|c|c|c|c|c|c|}
\hline $\begin{array}{l}\text { WFS } \rightarrow \\
\text { Instrument/ } \\
\text { AO-module/ } \\
\text { AO-mode } \downarrow\end{array}$ & $\begin{array}{c}\text { AO type: } \\
\mathrm{G}=\mathrm{GLAO} \\
\mathrm{L}=\mathrm{LTAO} \\
\mathrm{M}=\mathrm{MCAO} \\
\mathrm{S}=\mathrm{SCAO}\end{array}$ & $\begin{array}{c}\text { Type of WFS, } \\
\text { high-order (HO) } \\
\text { or low-order (LO) }\end{array}$ & $\begin{array}{l}\text { Wavefront } \\
\text { sensing } \\
\text { spectral } \\
\text { band }[\mu \mathrm{m}]\end{array}$ & $\begin{array}{c}\text { Wavefront } \\
\text { linear } \\
\text { spatial } \\
\text { sampling }[\mathrm{m}]\end{array}$ & $\begin{array}{c}\text { Wavefront } \\
\text { temporal } \\
\text { sampling }[\mathrm{Hz}]\end{array}$ & $\begin{array}{l}\text { Wavefront sensor } \\
\text { detector }\end{array}$ \\
\hline \multicolumn{7}{|c|}{ ELT } \\
\hline HARMONI $^{\mathrm{a}}$ & $\mathrm{S}$ & $1 \times$ Pyramid, HO & $0.5-1.0$ & 0.5 & 1000 & CCD220 \\
\hline HARMONI $^{\mathrm{b}}$ & $\mathrm{G}, \mathrm{L}$ & $6 \times \mathrm{SHS}, \mathrm{HO}$ & 0.589 & 0.5 & 500-1000 & $\operatorname{LVSM}^{\mathrm{h}}$ \\
\hline HARMONI $^{\mathrm{c}}$ & L & $1 \times \mathrm{SHS}, \mathrm{LO}$ & $1.4-1.8$ & 19.5 & 1000 & Saphira $^{\mathrm{i}}$ \\
\hline METIS $^{\mathrm{a}}$ & $\mathrm{S}$ & $1 \times$ Pyramid & $1.4-2.4$ & 0.5 & $1000-2000$ & Saphira $^{\mathrm{i}}$ \\
\hline MICADO $^{a}$ & $\mathrm{~S}$ & $1 \times$ Pyramid, $\mathrm{HO}$ & $0.45-0.9$ & $\lesssim 0.5$ & 1000 & CCD220 \\
\hline MAORYa & M & $3 \times$ Pyramid, $\mathrm{HO}$ & $0.6-0.8$ & $\lesssim 0.5$ & 500 & CCD220 \\
\hline MAORY ${ }^{b}$ & M & $6 \times \mathrm{SHS}, \mathrm{HO}$ & 0.589 & $\lesssim 0.5$ & 500 & LVSM $^{\mathrm{h}}$ \\
\hline MAORY $^{\mathrm{c}}$ & M & $3 \times \mathrm{SHS}, \mathrm{LO}$ & $1.5-1.8$ & 19.5 & 500 & Saphira $^{i}$ \\
\hline \multicolumn{7}{|c|}{ GMT } \\
\hline $\mathrm{SCAO}^{\mathrm{a}, \mathrm{d}}$ & $\mathrm{S}$ & $1 \times$ Pyramid, HO & $0.6-0.9$ & 0.28 & 1000 & CCD220 ${ }^{\mathrm{h}}$ \\
\hline $\mathrm{GLAO}^{\mathrm{a}, \mathrm{d}}$ & $\mathrm{G}$ & $4 \times \mathrm{SHS}, \mathrm{HO}$ & $0.5-1.0$ & $0.53-1.06$ & 200 & $\mathrm{EMCCD}^{\mathrm{m}}$ \\
\hline $\mathrm{LTAO}^{\mathrm{b}, \mathrm{d}}$ & $\mathrm{L}$ & $6 \times \mathrm{SHS}, \mathrm{HO}$ & 0.589 & 0.42 & 500 & NGSD $^{\mathrm{h}}$ \\
\hline OIWFS $^{c, d}$ & $\mathrm{~S}, \mathrm{~L}$ & $1 \times \mathrm{QC}, \mathrm{LO}$ & $2.03-2.37$ & 25.4 & 1000 & Saphira ${ }^{\mathrm{i}}$ \\
\hline OIWFS $^{c, d}$ & S, L & $1 \times \mathrm{SHS}, \mathrm{LO}$ & $1.65-1.8$ & 5.08 & 10 & Saphira ${ }^{\mathrm{i}}$ \\
\hline OIWFS $^{\mathrm{c}, \mathrm{d}}$ & $\mathrm{S}, \mathrm{L}$ & $1 \times \mathrm{SHS}, \mathrm{HO}$ & $1.17-1.33$ & 1.59 & 0.1 & Saphira ${ }^{\mathrm{i}}$ \\
\hline \multicolumn{7}{|c|}{ TMT } \\
\hline NFIRAOS $^{\mathrm{a}, \mathrm{e}}$ & $\mathrm{S}$ & $1 \times$ Pyramid, $\mathrm{HO}$ & $0.6-1.0$ & 0.31 & 800 & $\mathrm{MIT} / \mathrm{LL} \mathrm{CCD}^{\mathrm{j}}$ \\
\hline NFIRAOS ${ }^{\mathrm{b}, \mathrm{e}}$ & M & $6 \times \mathrm{SHS}, \mathrm{HO}$ & 0.589 & 0.5 & 800 & PC-CCD ${ }^{k}$ \\
\hline OIWFS $^{c, e}$ & M & $3 \times \mathrm{SHS}, \mathrm{LO}$ & $1.1-2.3$ & 15 & 800 & Hawaii-1/2RG or Saphiral \\
\hline ODGW $\mathrm{O}^{\mathrm{c}, \mathrm{e}}$ & M & 4 windows, $\mathrm{LO}$ & $0.84-2.4$ & 30 & 800 & IRIS detector \\
\hline
\end{tabular}

Notes: a Using NGSs, usually for SCAO modes or truth sensing for LGS observations.

${ }^{\mathrm{b}}$ Using sodium LGSs, usually for LTAO or MCAO modes.

${ }^{\mathrm{c}}$ Low-order NGS WFSs for LGS observations.

${ }^{\mathrm{d}}$ The GMT wavefront sensing architecture foresees integrated WFSs (SCAO, GLAO and LTAO), and on-instrument wavefront sensors (OIWFS).

eThe TMT wavefront sensing architecture foresees integrated WFSs (NFIRAOS: narrow field infrared AO system), on-instrument wavefront sensors (OIWFS), and instrument internal on-detector guide windows (ODGW). OIWFS and ODGW numbers are for the TMT instrument IRIS (Andersen et al., 2017).

${ }_{\mathrm{f}}$ ELT data taken from Correia et al. (2016); Neichel et al. (2016); Hippler et al. (2018); Sereni (2017); Clénet et al. (2016); Esposito et al. (2015). GMT numbers taken from Bouchez et al. (2014); Pinna et al. (2014); van Dam et al. (2014). TMT numbers taken from Boyer \& Ellerbroek (2016); Boyer (2017); Dunn et al. (2016); Kerley et al. (2016); Herriot et al. (2014, 2017).

gDowning et al. (2016).

${ }^{\mathrm{h}}$ Teledyne $\mathrm{e} 2 \mathrm{v}$ large sensor visible module (LSVM) and other sensors for AO (Jorden et al., 2017). For the natural guide star detector (NGSD) device see also Reyes-Moreno et al. (2016).

${ }^{\mathrm{i}}$ Atkinson et al. (2016) an references therein.

${ }^{\mathrm{j}} \mathrm{MIT} /$ Lincoln labs. $256 \times 256$ CCD. ${ }^{\mathrm{k}} \mathrm{MIT} /$ Lincoln labs. polar coordinate CCD (Adkins, 2012).

${ }^{l}$ TMT intention for OIWFS/ODGW (Boyer \& Ellerbroek, 2016). ${ }^{\mathrm{m}}$ Andor or Raptor EMCCD (Bouchez, 2017).

- calculate the wavefront slopes from calibrated pixel data,

- reconstruct the wavefront using for example a matrix-vector multiplication (MVM),

- calculate the new shapes and positions of the wavefront corrections devices including tasks like vibrations control, DM saturation management, etc.,
- send control commands to the wavefront correction devices,

is rather short and of the order of 0.2-2 milliseconds (Gratadour et al., 2016; Kerley et al., 2016; Bouchez et al., 2014). This "low latency" requirement defines the control bandwidth of the AO system as well as the stability and robustness of the closed-loop 


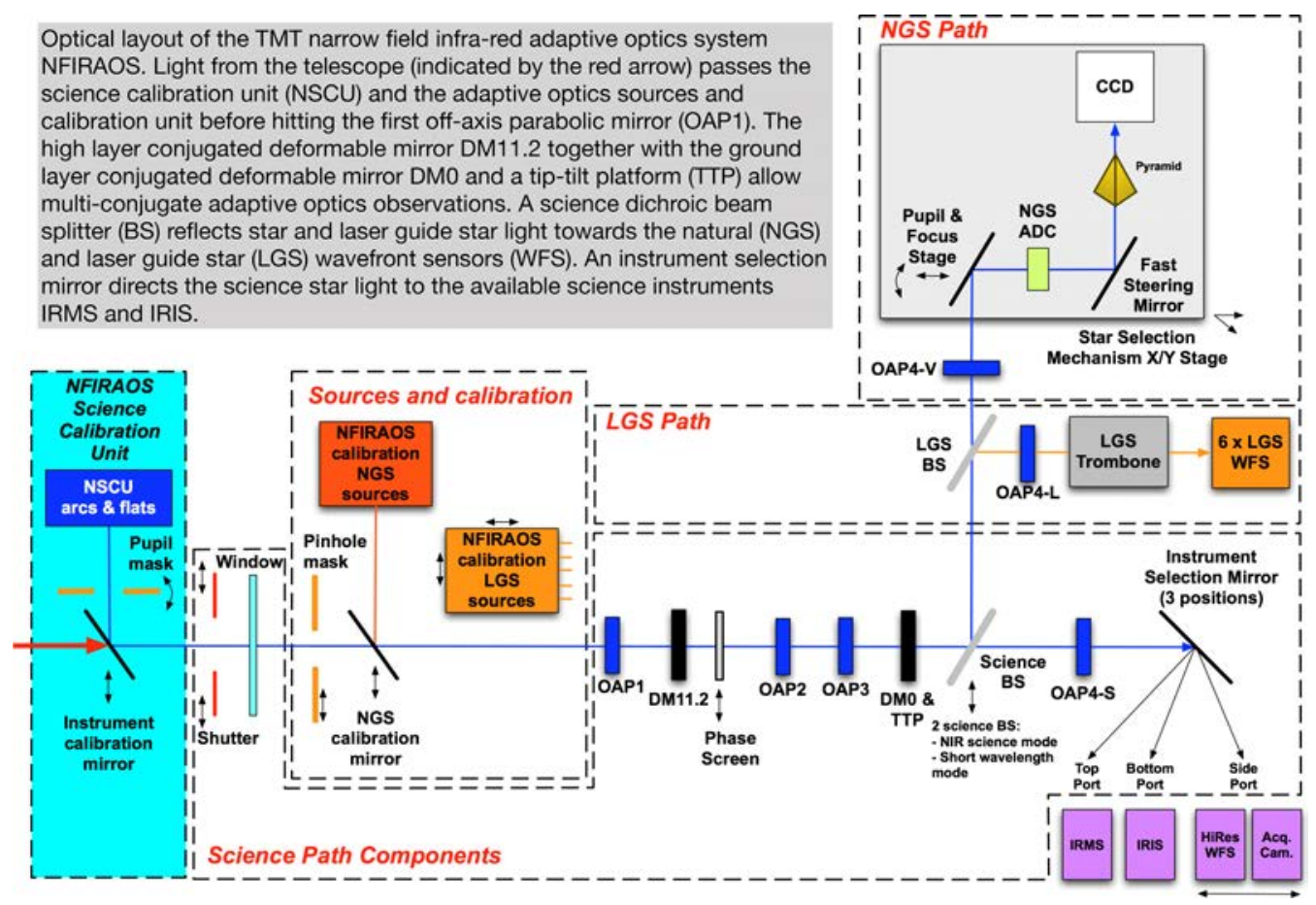

Fig. 7. Optical layout of the TMT's MCAO unit NFIRAOS. Picture without gray text box taken from (TMT, 2018a).

system. Looking at the matrix-vector multiplication, one of the most time consuming operations in this list, year 2016 off-the-shelf hardware is compliant with the latency requirements. Exemplary performance tests using Intel's Xeon Phi (Knights
Landing, KNL) CPU with 68 cores already shows satisfactory results as shown in Fig. 8.

A schematic and simplified view of the real-time control system of the ELT instrument METIS is shown in Fig. 9. The core of METIS SCAO real-time

Table 4. Adaptive DMs planned.

\begin{tabular}{|c|c|c|c|c|c|}
\hline $\begin{array}{l}\text { DM characteristics } \rightarrow \\
\text { Telescope location } \downarrow\end{array}$ & $\begin{array}{l}\text { \# act., } \\
\text { \# seg. }\end{array}$ & $\begin{array}{l}\text { act. } \\
\text { spacing } \\
{[\mathrm{m}]}\end{array}$ & $\begin{array}{l}\text { act. } \\
\text { time }^{\mathrm{c}} \\
{[\mathrm{ms}]}\end{array}$ & $\begin{array}{c}\text { act. } \\
\text { technology } \\
\mathrm{M}=\text { MEMS } \\
\mathrm{P}=\text { piezo stack } \\
\mathrm{V}=\text { voice coil }\end{array}$ & Comments \\
\hline GMT-M2 (ASM) ${ }^{\mathrm{d}}$ & 4704,7 & 0.36 & 1 & $\mathrm{~V}$ & $\begin{array}{l}\text { Based on the LBT adaptive secondary mirror, } \\
\text { optically conjugated to } 160 \mathrm{~m} \text { above ground. }\end{array}$ \\
\hline GMT OIWFS-DM ${ }^{\mathrm{e}}$ & $\geq 20 \times 20$ & $\leq 1.27$ & $0.5-2$ & $\mathrm{M}, \mathrm{P}, \mathrm{V}$ & $\begin{array}{l}\text { on-instrument DM for GMTIFS. Actuator technology } \\
\text { not yet decided. }\end{array}$ \\
\hline TMT-NFIRAOS-DM0 $0^{\mathrm{f}}$ & 3125,1 & $\lesssim 0.5$ & $\leq 1$ & $\mathrm{P}$ & Optically conjugated to the ground. \\
\hline TMT-NFIRAOS-DM11 ${ }^{\mathrm{f}}$ & 4548,1 & $\lesssim 0.5$ & $\leq 1$ & $\mathrm{P}$ & $\begin{array}{l}\text { Optically conjugated to } 11.2 \mathrm{~km} \text { height above } \\
\text { telescope. }\end{array}$ \\
\hline ELT-M $4^{g}$ & 5136,6 & 0.5 & 1 & $\mathrm{~V}$ & Optically conjugated to atmospheric ground layer. \\
\hline ELT-MAORY ${ }^{\mathrm{h}}$ & $\approx 500-1000$ & $1.5-2.0$ & & $\mathrm{~V}, \mathrm{P}$ & $\begin{array}{l}\text { ELT-M4 plus two DMs optically conjugated to } 4 \mathrm{~km} \\
\text { and } 14-16 \mathrm{~km} \text { in the atmosphere. Actuator } \\
\text { technology not yet decided (Lombini et al., 2016; } \\
\text { Pagès et al., 2016). }\end{array}$ \\
\hline
\end{tabular}

Notes: ${ }^{a}$ Total number of actuators (act.) and segments (seg.)

${ }^{\mathrm{b}}$ Actuator linear spacing over conjugated pupil $[\mathrm{m}]$. ${ }^{\mathrm{c}}$ Actuator settling time.

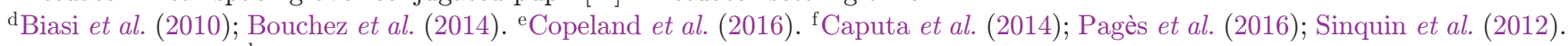

${ }^{\mathrm{g}}$ Biasi et al. (2016). ${ }^{\mathrm{h}}$ Lombini et al. (2016); Oberti et al. (2017). 


\section{S. Hippler}

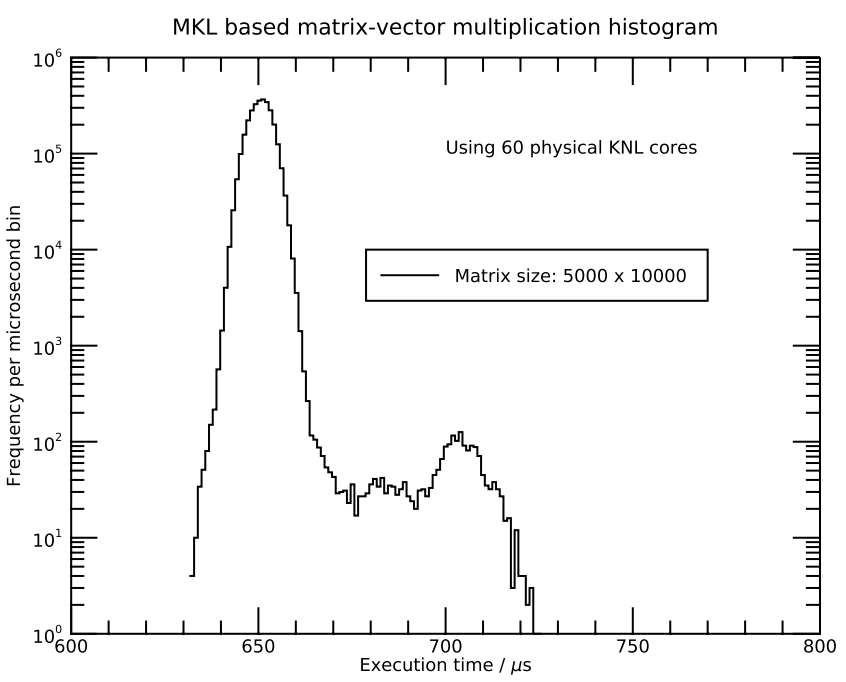

Fig. 8. Matrix-vector multiplication timing histogram using Intel's 1.4 GHz Xeon Phi 7250 (KNL) and Intel's Math Kernel Library (MKL). Matrix size: $5000 \times 10000$. Vector size 10000 with changing content. No. of samples: 3 million. Median execution time is $\sim 651$ microseconds with a standard deviation of $\sim 3$ microseconds.

control system (AO RTC) consists of a hard realtime controller (HRTC) and a soft real-time controller (SRTC). While the low-latency HRTC processes WFS data and generates control commands for the control systems of the correction devices, the soft real-time controller receives additional wavefront correction signals from the science focal planes (FPs) and their detector control systems (DCSs), respectively. Such additional wavefront corrections signals can for example compensate non-common path aberrations between the science and WFS light path, through adding slope offsets to the actual measured wavefront slopes. The generated correction commands can be either sent directly to the ELT's M4 and M5 local control systems (LCS) or through the ELT central control system (CCS). Further optical components within the commonpath of the METIS science and WFS channel are an optical de-rotator and the pupil stabilizer (pupil stabil.). Monitoring the pupil position can be performed on the SRTC and if necessary correction commands generated and sent to the pupil stabilization controller. The field selector inside the WFS channel is pre-set via the AO observation coordination system (OCS) and the pyramid WFS modulator has to be synchronized with the SCAO WFS detector control system. Wavefront control taking into account non-common path aberrations (NCPAs) is achieved through a communication channel between the science detectors and the SRTC. The AO function control system (FCS) completes the METIS SCAO control system.

To investigate real hardware options supporting all first-generation ELT instruments, the Green Flash project (Gratadour et al., 2016) aims in building a prototype/demonstrator designed to support all AO systems. After having investigated GPU clusters like the NVIDIA DGX-1 (Bernard

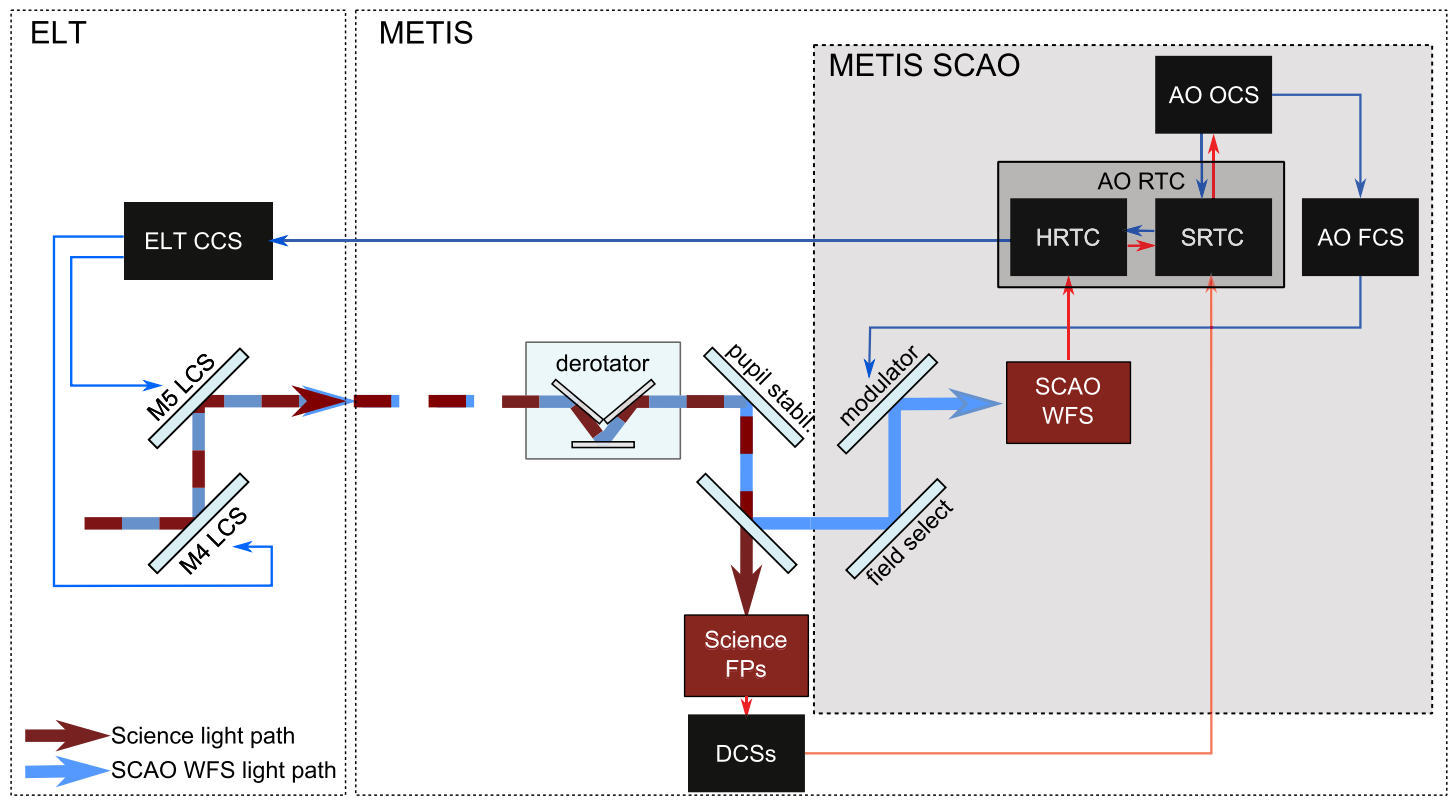

Fig. 9. Functional diagram of the METIS SCAO control system including non-common path aberrations control (Bertram et al., 2018). See text for details. 


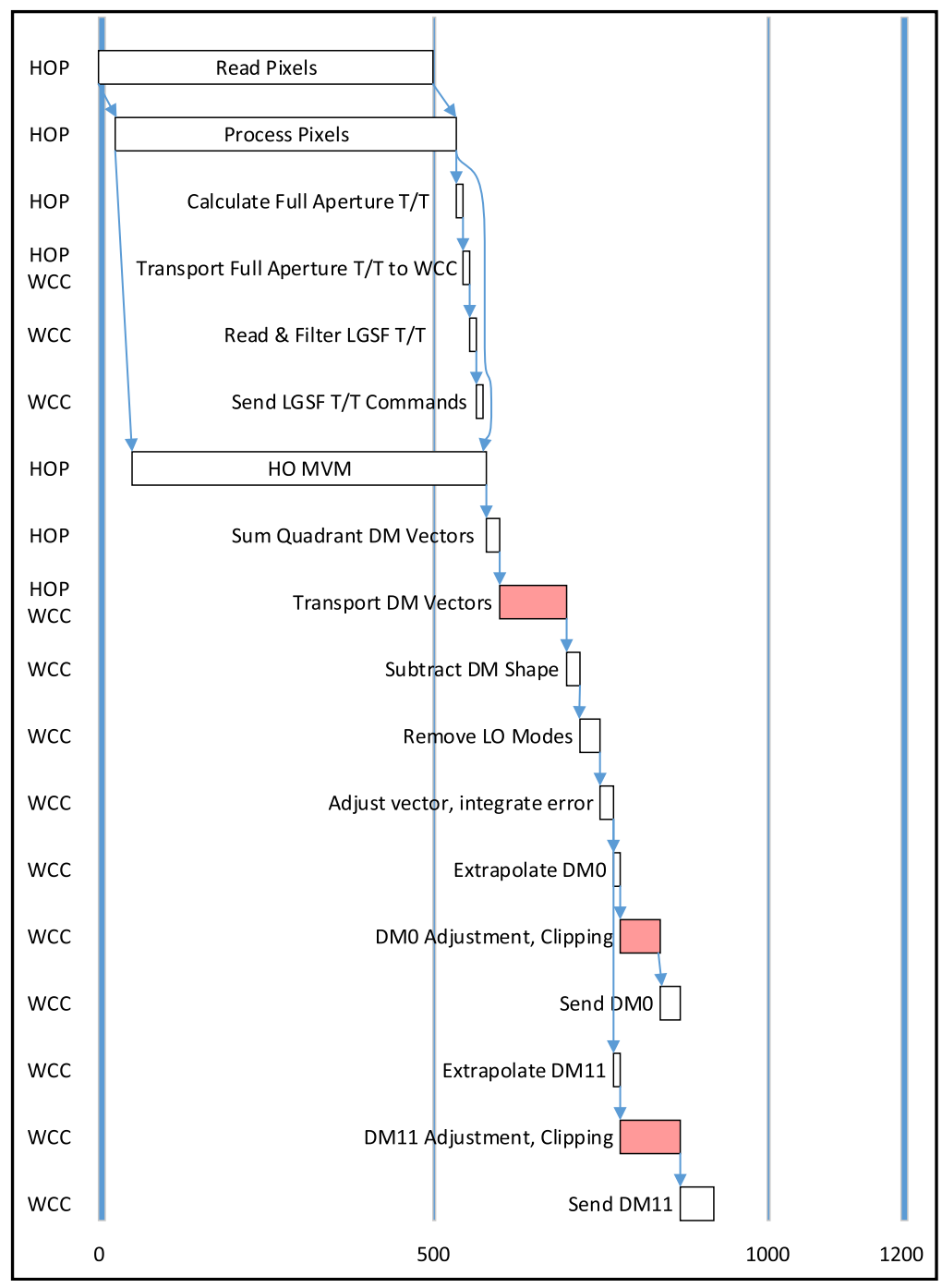

Fig. 10. Estimated real-time pipeline stage execution times of the TMT NFIRAOS RTC. Server name (left) and task name with box indicating the execution time in milliseconds. See text for details. Figure taken from Smith et al. (2016).

et al., 2017), Intel Xeon Phi multi-core devices (Jenkins et al., 2018), and a FPGA microserver (Green Flash Project, 2018), making the choice of a single solution (if necessary) remains open.

For the TMT's MCAO system NFIRAOS, more real-time tasks, compared to the METIS SCAO $\mathrm{RTC}$, are required to process the data acquired by up to six high-order WFSs. The sequence of the high-order AO data flow starts with pixel processing and wavefront reconstruction (HOP) for each WFS. A wavefront corrector control server (WCC) combines all measurements including low-order (LO) wavefront errors (full aperture NGS tip/tilt as well as LGS tip/tilt), and finally sends the correction signals to the high-order DMs DM0 and DM11 as well as to a tip-tilt stage (TTS), the LGS fast steering mirrors (FSM), and the telescope control system. The TMT RTC design allows to run the high-order pixel processing and wavefront reconstruction using a matrix-vector multiplication (MVM) almost in parallel. Estimated start and execution times for the various tasks are shown in Fig. 10.

The GMT's wavefront control system (WFCS) runs as an integral part of the telescope control

Table 5. Exemplary AO RTC HPC hardware planned or under study. Multiple devices are necessary.

\begin{tabular}{lccc}
\hline Telescope $\rightarrow$ & & & \\
Hardware $\downarrow$ & ELT & TMT & GMT \\
\hline CPUs & Intel Xeon Phi (KNL) & Intel E7-8870 V4 & yes \\
GPUs & NVIDIA DGX-1 & no & yes \\
FPGAs & Arria 10 & no & no \\
\hline
\end{tabular}




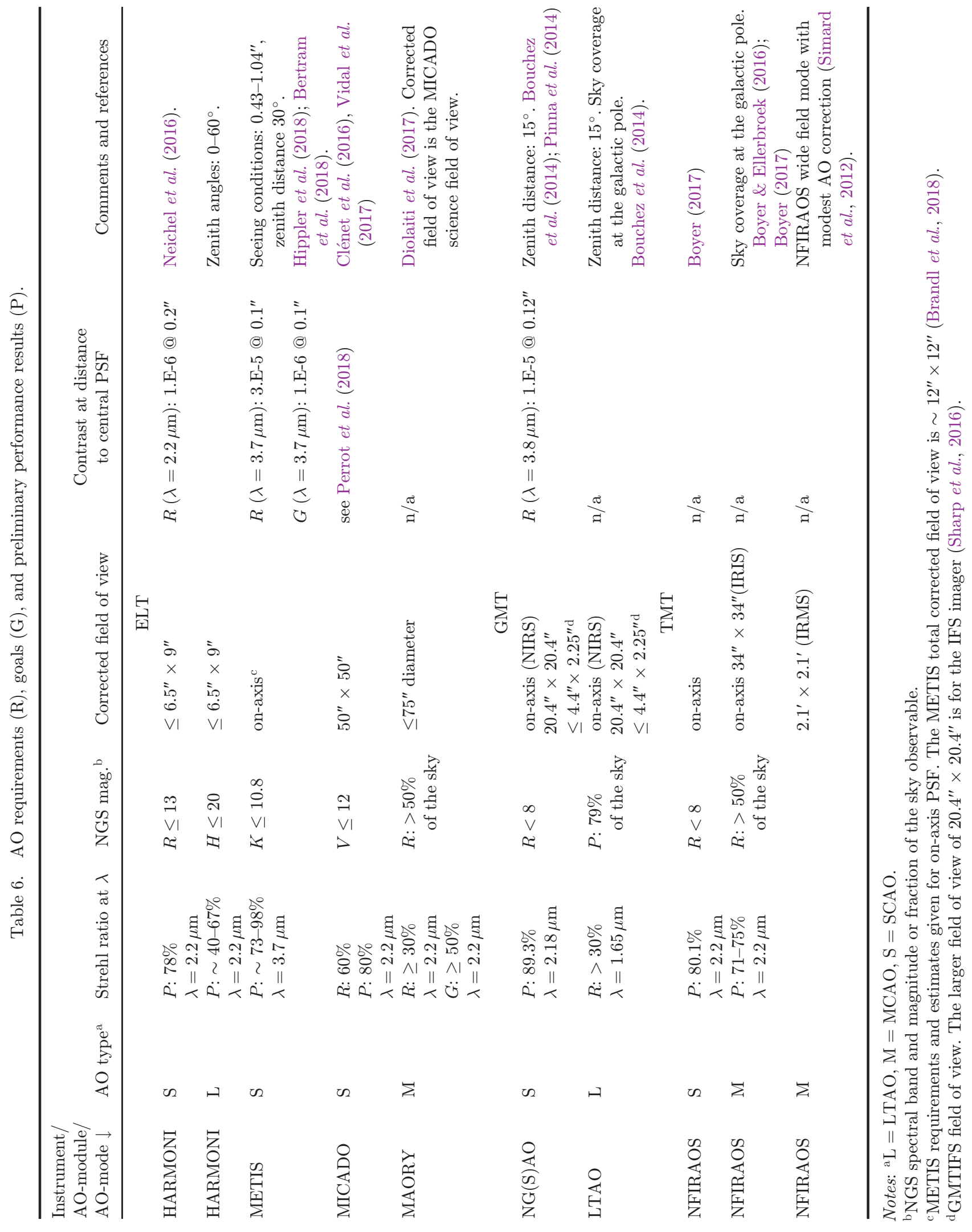


system Bouchez et al. (2014). The most demanding real-time requirements are set by the LTAO mode with a latency $\leq 200 \mu \mathrm{s}$. The GMT's baseline WFCS design foresees nine commodity servers, one slope processor for each of the six laser tomography WFSs, one node for communication with the adaptive secondary mirror, one node for communication with the on-instrument DM, and one master node for combining all reconstructed wavefronts.

Whether commercial off-the-shelf components and commodity servers can be used for the required high performance $\mathrm{AO}$ controllers is currently under investigation. Table 5 lists hardware components under consideration at the particular telescopes. The table brings into focus the high-performance computing (HPC) power only, while other hardware components like low-latency, high bandwidth networks (e.g. 10-100 Gbit-Ethernet, Infiniband, Omni-Path), sufficiently fast input/output channels (e.g. Camera Link, GigE Vision, sFPDP, USB3), and high throughput data storage are considered uncritical as it can be categorized as standard hardware.

\subsection{AO performance: Requirements and expectations}

Usually the scientific program determines the necessary minimum requirements for the measuring instrument. As an example, for the ELT METIS instrument various dedicated AO related top-level requirements influence the $\mathrm{AO}$ design. These are among others requirements on Strehl and contrast, for example, METIS shall deliver a minimum Strehl ratio of $60 \%$ (goal $80 \%$ ) in L-band $(\lambda=3.7 \mu \mathrm{m})$. For the contrast, METIS shall deliver a $5 \sigma$ contrast in L-band of $3 \times 10^{-5}$ (goal: $1 \times 10^{-6}$ ) at a distance to the central PSF of $5 \lambda / D$ (goal: $2 \lambda / D$ ).

Detailed end-to-end simulations are in the design phase the only way to figure out whether the design meets the requirements. Table 6 lists requirements on the $\mathrm{AO}$ for the instruments described in Table 1 and, where available, simulated performance numbers.

\section{High-Contrast Imaging Requirements on Adaptive Optics}

High-contrast imaging astronomy aims at uncovering faint structures and substellar objects very close to bright stars (an overview on this topic gives for example Oppenheimer \& Hinkley (2009)). Investigating distances close to the diffraction limit, i.e. 10-1000 mas, requires sophisticated AO installations, like XAO systems, that are able to spatially separate the light from, for instance, a host star and an orbiting planet.

A good measure to characterize the quality of an AO system is to look at the long and short exposure Strehl ratios it delivers. Together with the residual image motion, also for long and short exposures, one can use these static and dynamic performance data to estimate the imaging quality and the raw contrast delivered to the science channel. Per definition, the Strehl ratio defines the ratio of the measured $\mathrm{AO}$ corrected peak intensity of the point spread function (PSF) to the intensity measured or estimated of a perfectly imaged point spread function. If the delivered long exposure Strehl ratio at a wavelength of $3.7 \mu \mathrm{m}$ is $95 \%$, there is a fraction of $5 \%$ of intensity or energy in the halo of the stellar PSF. As the structure and the dynamic behavior of this un-controlled halo is unknown, it contributes strongly to the achievable contrast. This becomes clear if we look at the standard technique used in high-contrast imaging, where a stellar reference PSF is subtracted from the actual recorded stellar PSF.

In combination with a coronagraph, contrast values of point sources below $10^{-4}$ at angular separations of $5 \lambda / D$ on a $D=8 \mathrm{~m}$ telescope can be achieved. As shown in Fig. 11, using the AO instrument NACO at the $8 \mathrm{~m}$ VLT with an annular groove phase mask (AGPM), different contrast values can be achieved depending on the applied data reduction algorithm. The pure AO intensity profile of a standard star, which shows structures around the 3rd bright Airy ring and at the edge of the AO control radius, can be reduced by a factor of approx. 20 at an angular separation of 0.5 arcsec $(5 \lambda / D)$ with $\lambda=3.8 \mu \mathrm{m}$. The subtraction of the stellar PSF using the angular differential imaging (ADI, Marois et al. (2006)) method together with a principal components analysis (PCA, Gomez Gonzalez et al. (2016)) results in higher contrast values towards smaller angular separations. The rather flat contrast values at angular separations beyond the $\mathrm{AO}$ control radius indicate the highest contrast achievable at the smallest angular separations for this observation set-up. As the green curve in Fig. 11 shows, there are still about 2 orders of magnitude contrast improvements possible. 


\section{S. Hippler}

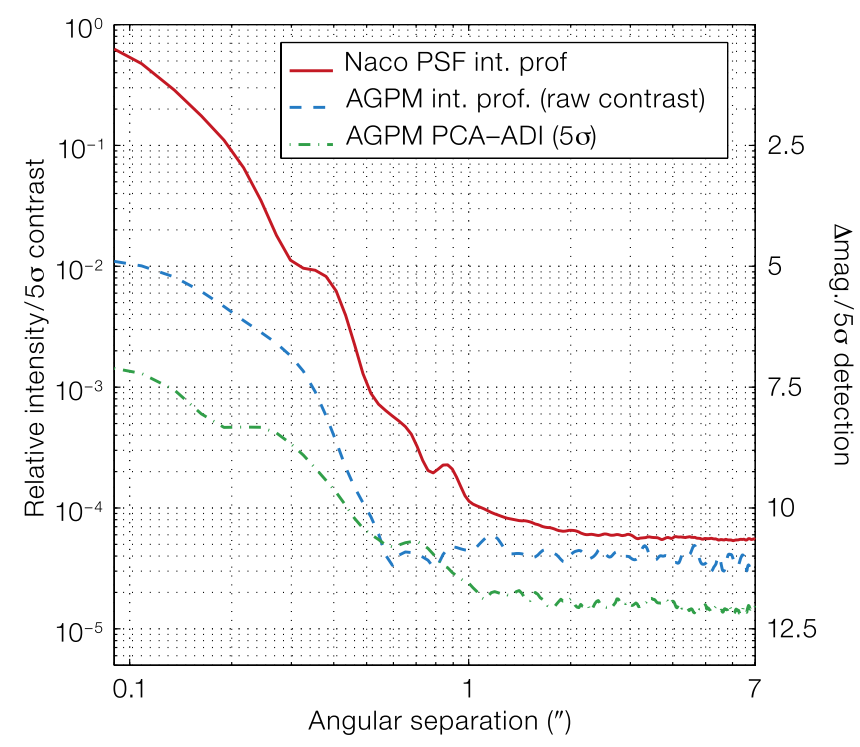

Fig. 11. VLT NACO coronagraphic observations of HD 123888 in L'-band $(\lambda=3.8 \mu \mathrm{m})$. Normalized azimuthally averaged relative intensity profiles and contrast curve on a log-log scale. The plain red curve shows the intensity profile of a typical saturated NACO L' PSF (similar brightness and exposure time). The blue dashed curve shows the AGPM intensity profile before PCA, demonstrating the instantaneous contrast gain provided by the coronagraph at all spatial frequencies within the AO control radius ( $\sim 0.7$ arcseconds). The green dash-dot curve presents the reduced PCA-ADI $5 \sigma$ detectability limits (40 frames, $800 \mathrm{~s}$, position angle difference $\approx 30^{\circ}$ ), taking both the coronagraph offaxis transmission and the PCA-ADI flux losses into account. Figures and caption taken from Mawet et al. (2013). See text for further explanations.

For that reason, it is important to understand in detail the contributions of the non-corrected aberrations to this regime.

As Mawet et al. (2012) point out, in particular low-order aberrations contribute to the contrast at angular separations between 1 and $4 \lambda / D$. One proposal to further improve the raw contrast behind an AO fed coronagraph is predictive control. Males \& Guyon (2018) show that the raw contrast for bright stars can be increased by more than three orders of magnitude at an angular separation from the PSF center of $1 \lambda / D$, for $\lambda=800 \mathrm{~nm}$ and $D=25.4 \mathrm{~m}$ (GMT case). This corresponds to a raw contrast improvement of $\sim 50$ at $\lambda=3.8 \mu \mathrm{m}$.

Another way to further reduce low-order aberrations is to increase the speed of the $\mathrm{AO}$ system in case there are enough photons available from the AO reference source. An exemplary case for the bright $(\mathrm{K}=4.5 \mathrm{mag})$ star 51 Eridani is shown in Fig. 12. Increasing the AO loop frequency from 1 to $2 \mathrm{kHz}$ improves the coronagraphic contrast by a factor $\approx 3$.

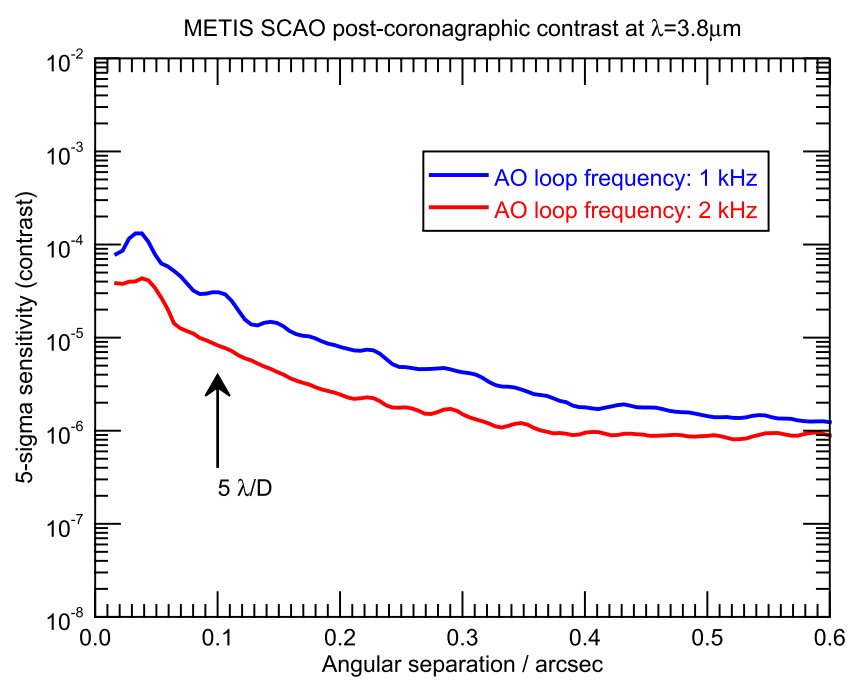

Fig. 12. ELT METIS $10 \mathrm{~s}$ simulated SCAO coronagraphic observations of the bright reference star 51 Eridani at $\lambda=3.8 \mu \mathrm{m}$ (Absil \& Carlomagno, 2018). This plot is to demonstrate that rather small changes of the long-exposure Strehl number from 0.978 at $1 \mathrm{kHz}$ AO loop speed to 0.984 at $2 \mathrm{kHz}$ AO loop speed results in a contrast enhancement of a factor $\sim 3$ at separation of $5 \lambda / D$. For comparison, in Fig. 11 this separation is at $\sim 0.5$ arcsec.

Guyon et al. (2012) have shown similar behavior for a perfect AO system on a $30 \mathrm{~m}$ class telescope, simulating wavefront sampling frequencies up to $100 \mathrm{kHz}$ and limiting the closed-loop servo

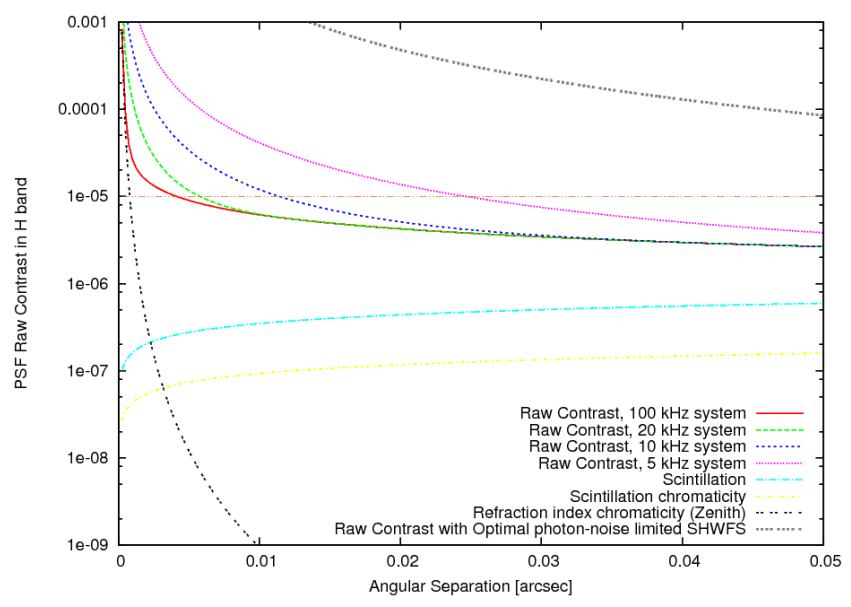

Fig. 13. PSF raw contrast of a $30 \mathrm{~m}$ telescope at $\lambda=1.6 \mu \mathrm{m}$ (H-band) vs. angular separation for wavefront sampling frequencies between 5 and $100 \mathrm{kHz}$. Wavefront sensing at $\lambda=0.8 \mu \mathrm{m}$ (I-band) using an ideal WFS. Shown are individual contributions to the contrast due to atmospheric scintillation, scintillation chromaticity, and atmospheric refraction chromaticity at zenith. A Shack-Hartmann wavefront sensor (SHWFS) not taking advantage of the $30 \mathrm{~m}$ telescope diffraction limit cannot deliver the performance of an ideal WFS. See text for further explanations. Figure taken from Guyon et al. (2012). 
Adaptive Optics for Extremely Large Telescopes

Table 7. Planned high-contrast instruments on ELT, GMT, and TMT.

\begin{tabular}{|c|c|c|c|}
\hline Telescope & Instrument name & Year & Comments \\
\hline ELT & MICADO, near-infrared wide-field imager & $\gtrsim 2024$ & 1st generation instr., Perrot et al. (2018). \\
\hline ELT & $\begin{array}{l}\text { METIS, mid-infrared ELT thermal imager and } \\
\text { spectrograph }\end{array}$ & $\gtrsim 2025$ & 1st generation instr., Kenworthy et al. (2016). \\
\hline ELT & $\begin{array}{l}\text { Planetary camera and spectrograph (PCS, also } \\
\text { called EPICS) }\end{array}$ & $\gtrsim 2028$ & 2nd generation instr., Kasper et al. (2013). \\
\hline GMT & $\begin{array}{l}\text { GMagAO-X, visible and near-infrared exoplanet } \\
\text { imager }\end{array}$ & $\gtrsim 2025$ & $\begin{array}{l}\text { 1st generation instr., Close et al. (2017); Bernstein } \\
\text { (2018) }\end{array}$ \\
\hline TMT & $\begin{array}{l}\text { b/blue Michi, a mid-infrared imager and } \\
\text { spectrometer }\end{array}$ & $\gtrsim 2030$ & $\begin{array}{l}\text { 2nd generation instr., Packham et al. (2012); } \\
\text { Packham (2017). }\end{array}$ \\
\hline TMT & Planetary Systems Instrument (PSI) & $\gtrsim 2030$ & $\begin{array}{l}\text { 2nd generation instr., successor of the Planet } \\
\text { Formation Imager (PFI) concept. TMT } \\
\text { (2018b); Dumas (2018). }\end{array}$ \\
\hline
\end{tabular}

latency to $0.1 \mathrm{~ms}$. As shown in Fig. 13, raw H-band PSF contrasts at small separations between 10 to 20 mas of $\approx 1$.e- 5 can be achieved.

This indicates that the stability of the AO controlled PSF is a critical factor for high-contrast imaging using coronagraphs and further postprocessing techniques. This is long known but the impact on contrast is striking. In particular PSF subtraction in combination with ADI requires careful execution as it can subtract extended structures like circumstellar disks Soummer et al. (2012). Measuring the reference PSF for post-processing PSF subtraction is a critical task in this context and requires the AO to perform as uniform as possible.

Table 7 provides an overview of the planned high-contrast instruments and the expected years of commissioning. Some of these instruments will also use the technology of high dispersion coronagraphy (HDC) not further explained here. A recent study on how exoplanets can be observed with HDC from the ground as well as with space telescopes can be found in Wang et al. (2017).

\section{Conclusions and Outlook}

AO technology has evolved enormously over the last 30 years. Designed as an anytime usable option for all ELTs currently in planning and construction, it will allow diffraction-limited observations in the near infrared range and at longer wavelengths. Expanding the use of $\mathrm{AO}$ in the visible spectrum remains challenging - a niche observing mode for the very bests nights as Close et al. (2017) suggest - , but we should keep our eyes on this.

All instruments under design for the ELT, GMT, and TMT can use AO, hence make use of the huge light collecting power and the maximum achievable angular resolution. LGS facilities push the AO sky coverage to levels well above $50 \%$.

Highly specialized instruments will further boost ground-based diffraction limited high-contrast imaging and characterization of exoplanets to unprecedented planet-star contrast ratios. The entire nearby Alpha Centauri system, including the planet Proxima Centauri b (Kreidberg \& Loeb, 2016) orbiting in the habitable zone, has received strong attraction in the science community, accompanied by an intense public attention. The pure imagination to find nearby Earth-like planets in habitable zones has triggered ideas to fly there for further investigation (Popkin, 2017).

\section{Acknowledgments}

The author would like to thank his colleagues Markus Feldt, Dietrich Lemke, and Kalyan Radhakrishnan for their advice and comments. Special thanks to the anonymous reviewer for his/her very valuable comments and suggestions to improve the quality of this review.

The author also thanks the following organizations and journals for allowing him to reproduce the figures listed below in this publication. The International Society for Optics and Photonics (SPIE) for Figs. 1, 6 and 9, the Monthly Notices of the Royal Astronomical Society for Fig. 3, the ESO for Fig. 2 and the background of Fig. 4, the Giant Magellan Telescope Organization (GMTO) for Fig. 5, the Thirty Meter Telescope International Observatory (TIO) for Fig. 7, and The Messenger for Fig. 11. 


\section{S. Hippler}

\section{References}

Absil, O. \& Carlomagno, B. [2018] "METIS coronagraphic simulations, March 2, 2018," personal communication.

Adkins, S. M. [2012] "Measured performance of the prototype polar coordinate CCD array," Proc. SPIE 8447, 84470R, doi: $10.1117 / 12.926474$.

Akiyama, M., Oya, S., Ono, Y. H. et al. [2014] "TMT-AGE: Wide field of regard multi-object adaptive optics for TMT," Proc. SPIE 9148, 914814, doi: 10.1117/12.2056320.

Ammons, S. M., Johnson, L., Laag, E. A. et al. [2008] "Laboratory demonstrations of multi-object adaptive optics in the visible on a 10 meter telescope," Proc. SPIE 7015, 70150C, doi: 10.1117/12.790188.

Andersen, D., Dunn, J., Larkin, J. et al. [2017] "Acquisition and dithering with the tmt iris on-instrument wavefront sensor system," in Proc. Adaptive Optics for Extremely Large Telescopes 5 (AO4ELT5), doi: 10.26698/AO4ELT5.012.

Andersen, D. R., Jackson, K. J., Blain, C. et al. [2012] Publ. Astron. Soc. Pacific 124, 469, doi: 10.1086/665924.

Atkinson, D. E., Hall, D. N. B., Baker, I. M. et al. [2016] "Nextgeneration performance of SAPHIRA HgCdTe APDs," Proc. SPIE 9915, p. $99150 \mathrm{~N}$, doi: $10.1117 / 12.2234314$.

Babcock, H. W. [1953] Publ. Astrono. Soc. Pacific 65, 229, doi: 10.1086/126606.

Bernard, J., Gratadour, D., Lainée, M. et al. [2017] "A gpu based rtc for e-elt adaptive optics: Real time controller prototype," in Proc. Adaptive Optics for Extremely Large Telescopes 5 (AO4ELT5), doi: 10.26698/AO4ELT5.0176.

Bernstein, R. A. [2018] "An introduction to the GMT: Telescope \& first generation instruments (Lecture 1)," in XVIII IAG/USP Advanced School on Astrophysics. GMT: Science and Instrumentation, Feb 26-Mar 1, 2018, São Paulo, Brazil.

Bertram, T., Absil, O., Bizenberger, P. et al. [2018] Proc. SPIE 10703, 1070314, doi: 10.1117/12.2313325.

Beuzit, J.-L., Feldt, M., Mouillet, D. et al. [2006] "A planet finder instrument for the VLT," in IAU Colloq. 200: Direct Imaging of Exoplanets: Science $\&$ Techniques, eds. Aime, C. \& Vakili, F. (Cambridge University Press), pp. 317-322, doi: $10.1017 /$ S1743921306009537.

Beuzit, J.-L., Hubin, N., Gendron, E. et al. [1994] "ADONIS: A user-friendly adaptive optics system for the ESO 3.6-m telescope," Proc. SPIE 2201, 955, doi: 10.1117/12.176129.

Biasi, R., Manetti, M., Andrighettoni, M. et al. [2016] "E-ELT M4 adaptive unit final design and construction: A progress report," Proc. SPIE 9909, 99097Y, doi: 10.1117/12.22 34735.

Biasi, R., Veronese, D., Andrighettoni, M. et al. [2010] "GMT adaptive secondary design," Proc. SPIE 7736, 77363O, doi: $10.1117 / 12.857983$.

Bonaccini Calia, D., Hackenberg, W., Holzlöhner et al. [2014] Adv. Opti. Technol. 3, 345, doi: 10.1515/aot-2014-0025.

Bouchez, A. [2017] "Giant Magellan Telescope wavefront control development status," in Adaptive Optics for Extremely Large Telescopes 5 (AO4ELT5), Jun 25-30, 2017, Tenerife, Spain.

Bouchez, A. H., Acton, D. S., Biasi, R. et al. [2014] "The Giant Magellan Telescope adaptive optics program," Proc. SPIE 9148, 91480W, doi: 10.1117/12.2057613.

Boyer, C. [2017] "Adaptive optics program update at TMT," Proc. Adaptive Optics for Extremely Large Telescopes 5 (AO4ELT5), doi: 10.26698/AO4ELT5.0131.
Boyer, C. \& Ellerbroek, B. [2016] "Adaptive optics program update at TMT," Proc. SPIE 9909, 990908, doi: 10.1117/ 12.2232945 .

Brandl, B. R., Agócs, T., Aitink-Kroes, G. et al. [2016] "Status of the mid-infrared E-ELT imager and spectrograph METIS," Proc. SPIE 9908, 990820, doi: 10.1117/12.2233974.

Brandl, B. R., Quanz, S., Snellen, I. et al. [2018] "The Mid-IR ELT Imager and Spectrograph (METIS) and its Science Goals in the Context of AKARI," in The Cosmic Wheel and the Legacy of the AKARI Archive: From Galaxies and Stars to Planets and Life, eds. Ootsubo, T., Yamamura, I., Murata, K. \& Onaka, T. (Japan Aerospace Exploration Agency), pp. 41-47.

Campbell, M. A., Evans, C. J., Mackey, A. D. et al. [2010] MNRAS 405, 421, doi: 10.1111/j.1365-2966.2010.16447.x.

Caputa, K., Atwood, J., Herriot, G. et al. [2014] "The 384channel prototype of DM Electronics for ELT AO systems," Proc. SPIE 9148, 914844, doi: 10.1117/12.2056580.

Clénet, Y., Buey, T., Rousset, G. et al. [2016] "Joint MICADOMAORY SCAO mode: Specifications, prototyping, simulations and preliminary design," Proc. SPIE 9909, 99090A, doi: $10.1117 / 12.2231192$.

Close, L., Males, J., Guyon, O. et al. [2017] "Current visible light ao systems and science with magao and a look to elts," in Adaptive Optics for Extremely Large Telescopes 5 (AO4ELT5), Jun 25-30, 2017, Tenerife, Spain.

Copeland, M., Price, I., Rigaut, F. et al. [2016] "GMTIFS: Deformable mirror environmental testing for the on-instrument wavefront sensor," Proc. SPIE 9909, 990980, doi: $10.1117 / 12.2231550$.

Correia, C. M., Neichel, B., Conan, J.-M. et al. [2016] "Natural guide-star processing for wide-field laser-assisted AO systems," Proc. SPIE 9909, 99094H, doi: 10.1117/12.223 2918.

Currie, T., Guyon, O., Tamura, M. et al. [2017] ApJL 836, L15, doi: 10.3847/2041-8213/836/1/L15.

Davidge, T. J., Andersen, D. R., Lardière, O. et al. [2015] ApJ 811, 133, doi: 10.1088/0004-637X/811/2/133.

Davies, R. \& Kasper, M. [2012] Annu. Rev. Astron. Astrophys. 50, 305, doi: 10.1146/annurev-astro-081811-125447.

Davies, R., Schubert, J., Hartl, M. et al. [2016] "MICADO: First light imager for the E-ELT," Proc. SPIE 9908, 99081Z, doi: 10.1117/12.2233047.

Davies, R. I., Hackenberg, W., Ott, T. et al. [1999] A $\mathcal{E} A S$ 138, 345, doi: 10.1051/aas:1999278.

DePoy, D. L., Allen, R., Barkhouser, R. et al. [2012] "GMACS: A wide field, multi-object, moderate-resolution, optical spectrograph for the Giant Magellan Telescope," Proc. SPIE 8446, 84461N, doi: 10.1117/12.926186.

Diolaiti, E., Ciliegi, P., Abicca, R. et al. [2016] "MAORY: Adaptive optics module for the E-ELT," Proc. SPIE 9909, 99092D, doi: 10.1117/12.2234585.

Diolaiti, E., Ciliegi, P., Abicca, R. et al. [2017] "On the road to the preliminary design review of the MAORY adaptive optics module for ELT," in Proc. Adaptive Optics for Extremely Large Telescopes 5 (AO4ELT5), doi: 10.26698/ AO4ELT5.0169.

D'Orgeville, C., Bouchez, A., Conan, R. et al. [2013] "GMT Laser Guide Star Facility," in Proc. Third AO4ELT Conf., eds. Esposito, S. \& Fini, L., p. 14, doi: 10.12839/AO4ELT3.13119.

d'Orgeville, C. \& Fetzer, G. J. [2016] "Four generations of sodium guide star lasers for adaptive optics in astronomy and 
space situational awareness," Proc. SPIE 9909, 99090R, doi: $10.1117 / 12.2234298$.

Downing, M., Casali, M., Finger, G. et al. [2016] "AO WFS detector developments at ESO to prepare for the E-ELT," Proc. SPIE 9909, 990914, doi: 10.1117/12.2232504.

Dumas, C. [2018] "The Thirty-Meter-Telescope project update, science in the 2020s," in Decadal Survey Community Planning Workshop, Feb 20-21, 2018, Tucson, USA.

Dunn, J., Andersen, D., Chapin, E. et al. [2016] "The Infrared Imaging Spectrograph (IRIS) for TMT: Multi-tiered wavefront measurements and novel mechanical design," Proc. SPIE 9908, 9908A9, doi: 10.1117/12.2234030.

ESO [2017] "Cutting-edge adaptive optics facility sees first light," https://www.eso.org/public/news/eso1724/

ESO [2018] "The ELT instruments," http://www.eso.org/ public/teles-instr/elt/

Esposito, S., Agapito, G., Antichi, J. et al. [2015] Mem. Soc. Astron. Ital. 86, 446.

Esposito, S., Mesa, D., Skemer, A. et al. [2013] A $\because A$ 549, A52, doi: 10.1051/0004-6361/201219212.

Esposito, S., Riccardi, A., Fini, L. et al. [2010] "First light AO (FLAO) system for LBT: Final integration, acceptance test in Europe, and preliminary on-sky commissioning results," Proc. SPIE 7736, 773609, doi: 10.1117/12.858194.

Esposito, S., Tozzi, A., Puglisi, A. et al. [2006] "First light AO system for LBT: Toward on-sky operation," Proc. SPIE 6272, 62720A, doi: 10.1117/12.673509.

Feldt, M., Gratton, R., Hippler, S. et al. [2007] "VI," The CHEOPS Project: Characterizing Exoplanets by Opto-infrared Polarimetry and Spectroscopy (Springer-Verlag), p. 261, doi: 10.1007/978-3-540-39756-4_69.

Fugate, R. Q., Wopat, L. M., Fried, D. L. et al. [1991] Nature 353, 144, doi: 10.1038/353144a0.

Fusco, T., Rousset, G., Beuzit, J.-L. et al. [2005] "Conceptual design of an extreme AO dedicated to extra-solar planet detection by the VLT-Planet Finder instrument," Proc. SPIE 5903, 59030K, doi: 10.1117/12.616518.

Fusco, T., Rousset, G., Sauvage, J.-F. [2006] Opt. Exp. 14, 7515, doi: 10.1364/OE.14.007515.

Fusco, T., Sauvage, J.-F., Petit, C. et al. [2014] "Final performance and lesson-learned of SAXO, the VLT-SPHERE extreme AO: From early design to on-sky results," Proc. SPIE 9148, 91481U, doi: 10.1117/12.2055423.

Gendron, E., Cuby, J., Rigaut, F. et al. [1991] "Come-on project - an upgrade of the come-on adaptive optics prototype system," Proc. SPIE 1542, 297, doi: 10.1117/12.48818.

Gendron, E., Vidal, F., Brangier, M. et al. [2011] A $\& A$ 529, L2, doi: 10.1051/0004-6361/201116658.

Gilmozzi, R., Delabre, B., Dierickx, P. et al. [1998] "Future of filled aperture telescopes: Is a 100-m feasible?" Proc. SPIE 3352, 778, doi: 10.1117/12.319284.

GMTO [2013] "GMT system level preliminary design review," Section 6, https://www.gmto.org/resources/slpdr/.

Gomez Gonzalez, C. A., Absil, O., Absil, P.-A. et al. [2016] AESA 589, A54, doi: 10.1051/0004-6361/201527387.

Graham, J. R., Macintosh, B., Doyon, R. et al. [2007] arXiv: 0704.1454 .

Gratadour, D., Dipper, N., Biasi, R. et al. [2016] "Green FLASH: Energy efficient real-time control for AO," Proc. SPIE 9909, 99094I, doi: 10.1117/12.2232642.
Gratton, R., Feldt, M., Schmid, H. M. et al. [2004] "The science case of the CHEOPS planet finder for VLT," Proc. SPIE 5492, 1010, doi: 10.1117/12.551417.

Green Flash Project [2018] "Green flash, high performance computing for real-time science, prototype review 06/04/ 2018 Paris," http://greenflash-h2020.eu/sites/green-flash/ IMG/pdf/gflash-fdr-mic-wp3.pdf.

Guyon, O., Martinache, F., Cady, E. J. et al. [2012] "How ELTs will acquire the first spectra of rocky habitable planets," Proc. SPIE 8847, 84471X, doi: 10.1117/12.927181.

Guyon, O., Martinache, F., Garrel, V. et al. [2010] "The Subaru coronagraphic extreme AO (SCExAO) system: Wavefront control and detection of exoplanets with coherent light modulation in the focal plane," Proc. SPIE 7736, 773624, doi: $10.1117 / 12.857878$.

Hackenberg, W., Eckart, A., Davies, R. I. et al. [2000] $A \mathscr{E} A$ 363, 41 .

Hardy, J. W., Lefebvre, J. E. \& Koliopoulos, C. L. [1977] J. Opt. Soc. Am. (1917-1983) 67, 360.

Hart, M., Milton, N. M., Baranec, C. et al. [2010] Nature 466 , 727, doi: 10.1038/nature09311.

Herriot, G., Andersen, D., Atwood, J. et al. [2014] "NFIRAOS: First facility AO system for the Thirty Meter Telescope," Proc. SPIE 9148, 914810, doi: 10.1117/12.2055525.

Herriot, G., Andersen, D., Atwood, J. et al. [2017] "NFIRAOS," in Proc. Adaptive Optics for Extremely Large Telescopes 5 (AO4ELT5), doi: 10.26698/AO4ELT5.0129.

Hippler, S., Feldt, M., Bertram, T. et al. [2018] Experimental Astronomy, doi: 10.1007/s10686-018-9609-y.

Jacoby, G. H., Bernstein, R., Bouchez, A. et al. [2016] "Instrumentation progress at the Giant Magellan Telescope project," Proc. SPIE 9908, 99081U, doi: 10.1117/ 12.2232809 .

Jaffe, D. T., Barnes, S., Brooks, C. et al. [2016] "GMTNIRS: Progress toward the Giant Magellan Telescope near-infrared spectrograph," Proc. SPIE 9908, 990821, doi: 10.1117/ 12.2232994

Jenkins, D. R., Basden, A. \& Myers, R. M. [2018] MNRAS 478, 3149, doi: 10.1093/mnras/sty1310.

Jorden, P., Bourke, D., Cassidy, R. et al. [2017] "Teledyne e2v sensors for adaptive optics: Wavefront sensing on elts: High rate, large format, and low noise," in Proc. Adaptive Optics for Extremely Large Telescopes 5 (AO4ELT5), doi: 10.26698/AO4ELT5.0009.

Kasper, M., Verinaud, C. \& Mawet, D. [2013] "Roadmap for PCS, the Planetary Camera and Spectrograph for the EELT," in Proc. Third AO4ELT Conf., eds. Esposito, S. \& Fini, L., p. 8, doi: 10.12839/AO4ELT3.12804.

Kenworthy, M. A., Absil, O., Agócs, T. et al. [2016] "Highcontrast imaging with METIS," Proc. SPIE 9908, 9908A6, doi: $10.1117 / 12.2233655$.

Kerley, D., Smith, M., Dunn, J. et al. [2016] "Thirty Meter Telescope (TMT) narrow field infrared adaptive optics system (NFIRAOS) real-time controller preliminary architecture," Proc. SPIE 9913, 99134J, doi: 10.1117/ 12.2233709 .

Kopf, T., Dietzel, O., Dargatz, B. et al. [2017] "SWAP DM: Preliminary design and schematics of a DM for extreme adaptive optics," in Proc. Adaptive Optics for Extremely Large Telescopes 5 (AO4ELT5), doi: 10.26698/AO4ELT5.0117. 


\section{S. Hippler}

Kreidberg, L. \& Loeb, A. [2016] ApJL 832, L12, doi: 10.3847/ 2041-8205/832/1/L12.

La Penna, P., Aller Carpentier, E., Argomedo, J. et al. [2016] "AOF: Standalone test results of GALACSI," Proc. SPIE 9909, 99092Z, doi: 10.1117/12.2232984.

Laag, E. A., Ammons, S. M., Gavel, D. T. et al. [2008] J. Opt. Soc. Am. A 25, 2114, doi: 10.1364/JOSAA.25.002114.

Lamb, M., Venn, K., Andersen, D. et al. [2017] MNRAS 465, 3536, doi: 10.1093/mnras/stw2865.

Lardière, O., Andersen, D., Blain, C. et al. [2014] "Multi-object adaptive optics on-sky results with Raven," Proc. SPIE 9148, 91481G, doi:10.1117/12.2055480.

Larkin, J. E., Moore, A. M., Wright, S. A. et al. [2016] "The infrared imaging spectrograph (IRIS) for TMT: Instrument overview," Proc. SPIE 9908, 99081W, doi: 10.1117/12.2232212.

Lee, K.-D., Kim, Y.-S., Kim, H.-S., Lee, C. H. \& Lee, W. G. [2017] Publ. Astron. Soc. Pacific 129, 095001, doi: 10.1088/ 1538-3873/aa76bd.

Leibundgut, B., Bacon, R., Jaffé, Y. L. et al. [2017] The Messenger 170, 20.

Li, M., Wei, K., Tang, J. et al. [2016] "The progress of TMT Laser Guide Star Facility," Proc. SPIE 9909, 99095Q, doi: 10.1117/12.2231987.

Lloyd-Hart, M., Brusa, G., Wildi, F. P. et al. [2003] "Lessons learned from the first adaptive secondary mirror," Proc. SPIE 5169, 79, doi: 10.1117/12.507316.

Lombini, M., De Rosa, A., Ciliegi, P. et al. [2016] "Optical design of the post-focal relay of MAORY," Proc. SPIE 9908, 9908AB, doi: 10.1117/12.2234569.

Lozi, J., Guyon, O., Jovanovic, N. et al. [2017] "SCExAO: Lessons learned for a future TMT instrument," in Proc. Adaptive Optics for Extremely Large Telescopes 5 (AO4ELT5), doi: 10.26698/AO4ELT5.0080.

Lozi, J., Guyon, O., Jovanovic, N. et al. [2016] "Characterizing and mitigating vibrations for SCExAO," Proc. SPIE 9909, 99090J, doi: 10.1117/12.2233040.

Macintosh, B., Graham, J., Palmer, D. et al. [2006a] "The Gemini Planet Imager," Proc. SPIE 6272, 62720L, doi: $10.1117 / 12.672430$.

Macintosh, B., Graham, J. R., Barman, T. et al. [2015] Science 350, 64, doi: 10.1126/science.aac5891.

Macintosh, B., Troy, M., Doyon, R. et al. [2006b] "Extreme adaptive optics for the Thirty Meter Telescope," Proc. SPIE 6272, 62720N, doi: 10.1117/12.672032.

Madec, P.-Y. [2012] "Overview of deformable mirror technologies for adaptive optics and astronomy," Proc. SPIE 8447, 844705, doi: 10.1117/12.924892.

Maire, A.-L., Bonnefoy, M., Ginski, C. et al. [2016] A\&A 587, A56, doi: 10.1051/0004-6361/201526594.

Males, J. R. \& Guyon, O. [2018] J. Astron. Telesc. Instrum. Syst. 4, 019001, doi: 10.1117/1.JATIS.4.1.019001.

Marchetti, E., Brast, R., Delabre, B. et al. [2007] The Messenger 129, 8 .

Marchetti, E., Brast, R., Delabre, B. et al. [2008] "MAD on sky results in star oriented mode," Proc. SPIE 7015, 70150F, doi: $10.1117 / 12.787240$.

Marin, E., Sivo, G., Garrel, V. et al. [2017] "Tracking the sodium layer altitude with GeMS in the era of NGS2," in Proc. Adaptive Optics for Extremely Large Telescopes 5 (AO4ELT5), doi: 10.26698/AO4ELT5.0090.
Marois, C., Lafrenière, D., Doyon, R. et al. [2006] ApJ 641, 556, doi: $10.1086 / 500401$.

Martinache, F. \& Guyon, O. [2009] "The Subaru Coronagraphic Extreme-AO Project" Proc. SPIE 7440, 74400O, doi: $10.1117 / 12.826365$.

Mawet, D., Absil, O., Girard, J. H. et al. [2013] The Messenger 152,8 .

Mawet, D., Pueyo, L., Lawson, P. et al. [2012] "Review of smallangle coronagraphic techniques in the wake of ground-based second-generation adaptive optics systems," Proc. SPIE 8442, 844204, doi: 10.1117/12.927245.

Meyer, E., Kürster, M., Arcidiacono, C., Ragazzoni, R \& Rix, H.-W. [2011] A\&A 532, A16, doi: 10.1051/0004-6361/ 201016053.

Milli, J., Kasper, M., Bourget, P. et al. [2018] "Low wind effect on VLT/SPHERE: Impact, mitigation strategy, and results," Proc. SPIE 10703, 107032A, doi: 10.1117/12.2311499.

Milli, J., Mawet, D., Mouillet, D., Kasper, M. \& Girard, J. H. [2016] "Adaptive optics in high-contrast imaging," in Astronomy at High Angular Resolution, eds. Boffin, H. M. J., Hussain, G., Berger, J.-P. \& Schmidtobreick, L. (Springer), p. 17, doi: 10.1007/978-3-319-39739-9_2.

Mobasher, B., Crampton, D. \& Simard, L. [2010] "An infrared multi-object spectrograph (IRMS) with adaptive optics for TMT: The science case," Proc. SPIE 7735, 77355P, doi: $10.1117 / 12.858061$.

Morris, T., Basden, A., Buey, T. et al. [2016] "Adaptive optics for MOSAIC: Design and performance of the wide(st)-field AO system for the E-ELT," Proc. SPIE 9909, 99091I, doi: $10.1117 / 12.2232411$.

Neichel, B., Fusco, T., Sauvage, J.-F. et al. [2016] "The adaptive optics modes for HARMONI: From Classical to Laser Assisted Tomographic AO," Proc. SPIE 9909, 990909, doi: $10.1117 / 12.2231681$.

Neichel, B., Rigaut, F., Vidal, F. et al. [2014] MNRAS 440, 1002, doi: 10.1093/mnras/stu403.

Oberti, S., Le Louarn, M., Diolaiti, E. et al. [2017] "MAORY design trade-off study: Tomography dimensioning," in Proc. Adaptive Optics for Extremely Large Telescopes 5 (AO4ELT5), doi: 10.26698/AO4ELT5.0162.

OIR-Laboratory-UCSD [2017] "IRIS, first light instrument for the Thirty Meter Telescope (TMT)," https://oirlab.ucsd. edu/IRIS.html.

Ono, Y. H., Correia, C. M., Lardière, O. et al. [2016] "On-sky MOAO performance evaluation of RAVEN," Proc. SPIE 9909, 990910, doi: 10.1117/12.2232321.

Oppenheimer, B. \& Hinkley, S. [2009] Ann. Rev. Astron. Astrophys. 47, 253, doi: 10.1146/annurev-astro-082708-101717.

Orban de Xivry, G., Rabien, S., Busoni, L. et al. [2016] "First on-sky results with ARGOS at LBT," Proc. SPIE 9909 , 990936, doi: $10.1117 / 12.2240094$.

Packham, C. [2017] "MICHI: A thermal-IR instrument for the TMT," in TMT: Beyond First Light, Nov 7-9, 2017, Mysore, India.

Packham, C., Honda, M., Richter, M. et al. [2012] "Key science drivers for MICHI: A mid-IR instrument concept for the TMT," Proc. SPIE 8446, 84467G, doi: 10.1117/12.924996.

Pagès, H., Antonini, T., Aribi, T. et al. [2016] "Developments of piezo deformable mirrors," Proc. SPIE 9909, 99097Z, doi: $10.1117 / 12.2232971$. 
Paufique, J., Madec, P.-Y., Kolb, J. et al. [2016] "GRAAL on the mountaintop," Proc. SPIE 9909, 99092H, doi: 10.1117/ 12.2232826 .

Perrot, C., Baudoz, P., Boccaletti, A. et al. [2018] "Design study and first performance simulation of the ELT/ MICADO focal plane coronagraphs," in Proc. Adaptive Optics for Extremely Large Telescopes 5 (AO4ELT5), doi: 10.26698/AO4ELT5.0159.

Pinna, E., Agapito, G., Quirós-Pacheco, F. et al. [2014] "Design and numerical simulations of the GMT Natural Guide star WFS," Proc. SPIE 9148, 91482M, doi: 10.1117/12.20 57059.

Popkin, G. [2017] Nat. News 542, 20.

Ragazzoni, R. [1999] A\&AS 136, 205, doi: 10.1051/ aas:1999207.

Ragazzoni, R., Marchetti, E. \& Rigaut, F. [1999] A\&GA 342, L53.

Ragazzoni, R., Marchetti, E. \& Valente, G. [2000] Nature 403, 54, doi: 10.1038/47425.

Reyes-Moreno, J., Downing, M. \& Di Lieto, N. [2016] "ESO adaptive optics NGSD/LGSD detector and camera controller for the E-ELT," Proc. SPIE 9909, 990962, doi: $10.1117 / 12.2232317$.

Riaud, P. [2012] A\&A 545, A25, doi: 10.1051/0004-6361/ 201219707.

Rigaut, F. [2002] "Ground Conjugate Wide Field Adaptive Optics for the ELTs," European Southern Observatory Conference and Workshop Proceedings, eds. Vernet, E., Ragazzoni, R., Esposito, S. \& Hubin, N., p. 11.

Rigaut, F., Neichel, B., Boccas, M. et al. [2014] MNRAS 437, 2361, doi: $10.1093 / \mathrm{mnras} / \mathrm{stt} 2054$.

Rousset, G., Fontanella, J. C., Kern, P., Gigan, P. \& Rigaut, F. [1990] A\&SA 230, L29.

Sereni, G. [2017] "Optical design of maory laser guide star objective," PhD thesis, Università di Bologna, Italy.

Sharp, R., Bloxham, G., Boz, R. et al. [2016] "GMTIFS: The Giant Magellan Telescope integral fields spectrograph and imager," Proc. SPIE 9908, 99081Y, doi: 10.1117/ 12.2231561 .

Simard, L., Crampton, D., Ellerbroek, B. \& Boyer, C. [2012] "The instrumentation program for the Thirty Meter Telescope," Proc. SPIE 8446, 84461F, doi: 10.1117/ 12.930500

Sinquin, J.-C., Bastard, A., Boyer, C. et al. [2012] "TMT DMs final design and advanced prototyping results at Cilas," Proc. SPIE 8447, 844706, doi: 10.1117/12.925313.

Smith, M., Kerley, D., Chapin, E. L. et al. [2016] "Thirty Meter Telescope narrow-field infrared adaptive optics system realtime controller prototyping results," Proc. SPIE 9909, 99094N, doi: 10.1117/12.2233711.

Soummer, R., Pueyo, L. \& Larkin, J. [2012] ApJL 755, L28, doi: $10.1088 / 2041-8205 / 755 / 2 / L 28$.
Szentgyorgyi, A., Baldwin, D., Barnes, S. et al. [2016] "The GMT-Consortium Large Earth Finder (G-CLEF): An optical Echelle spectrograph for the Giant Magellan Telescope (GMT)," Proc. SPIE 9908, 990822, doi: 10.1117/ 12.2233506 .

Thatte, N. A., Clarke, F., Bryson, I. et al. [2016] "The E-ELT first light spectrograph HARMONI: Capabilities and modes," Proc. SPIE 9908, 99081X, doi: 10.1117/12.2230629.

Thomas, S. J., Belikov, R. \& Bendek, E. [2015] "A method to directly image exoplanets in multi-star systems such as Alpha-Centauri," Proc. SPIE 9605, 96052G, doi: 10.1117/ 12.2188637 .

TMT [2017a] "Call for TMT instrumentation white papers," https://www.tmt.org/announcement/call-for-tmt-instrumentation-white-papers.

TMT [2017b] "TMT first-light instrument enters final design phase," https://www.tmt.org/news/tmt20171113.

TMT [2018a] "TMT adaptive optics," https://www.tmt.org/ page/adaptive-optics.

TMT [2018b] "TMT: Overview of instrument capabilities," https://www.tmt.org/page/instrument-overview.

TMT-UCR [2014] "IRMS, infrared multi-slit spectrograph for TMT," http://faculty.ucr.edu/ mobasher/irms/3.

van Dam, M. A., Bouchez, A. H. \& Conan, R. [2016] "Novel tiptilt sensing strategies for the laser tomography adaptive optics system of the GMT," Proc. SPIE 9909, 990965, doi: $10.1117 / 12.2231143$.

van Dam, M. A., Bouchez, A. H. \& McLeod, B. A. [2014] "Wide field adaptive optics correction for the GMT using natural guide stars," Proc. SPIE 9148, 914813, doi: 10.1117/ 12.2054154

van Dam, M. A., Sasiela, R. J., Bouchez, A. H. et al. [2006] "Angular anisoplanatism in laser guide star adaptive optics," Proc. SPIE 6272, 627231, doi: 10.1117/12.669737.

Vidal, F., Ferreira, F., Deo, V. et al. [2017] "End-to-end simulations for the micado-maory scao mode," in Proc. Adaptive Optics for Extremely Large Telescopes 5 (AO4ELT5), doi: 10.26698/AO4ELT5.0124.

Vidal, F., Gendron, E. \& Rousset, G. [2010] J. Opt. Soc. Am. A 27, A253, doi: 10.1364/JOSAA.27.00A253.

Vigan, A., Bonnefoy, M., Ginski, C. et al. [2016] A\& A 587, A55, doi: 10.1051/0004-6361/201526465.

Wang, J., Mawet, D., Ruane, G., Hu, R. \& Benneke, B. [2017] AJ 153, 183, doi: 10.3847/1538-3881/aa6474.

Wang, L., Andersen, D. \& Ellerbroek, B. [2012] Appl. Opt. 51, 3692, doi: 10.1364/AO.51.003692.

Wizinowich, P. [2012] "Progress in laser guide star adaptive optics and lessons learned," Proc. SPIE 8447, 84470D, doi: $10.1117 / 12.925093$.

Wizinowich, P., Acton, D. S., Shelton, C. et al. [2000] Publ. Astron. Soc. Pacific 112, 315, doi: 10.1086/316543. 